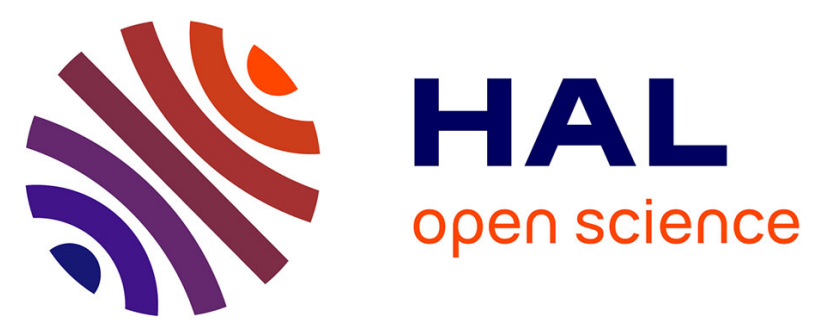

\title{
Heart rate variability in type 2 diabetes mellitus: A systematic review and meta- analysis
}

Thomas Benichou, Bruno Pereira, Martial Mermillod, Igor Tauveron, Daniela Pfabigan, Salwan Maqdasy, Frederic Dutheil

\section{- To cite this version:}

Thomas Benichou, Bruno Pereira, Martial Mermillod, Igor Tauveron, Daniela Pfabigan, et al.. Heart rate variability in type 2 diabetes mellitus: A systematic review and meta- analysis. PLoS ONE, 2018, 13 (4), 10.1371/journal.pone.0195166 . hal-01820031

\section{HAL Id: hal-01820031 \\ https://hal.science/hal-01820031}

Submitted on 21 Jun 2018

HAL is a multi-disciplinary open access archive for the deposit and dissemination of scientific research documents, whether they are published or not. The documents may come from teaching and research institutions in France or abroad, or from public or private research centers.
L'archive ouverte pluridisciplinaire HAL, est destinée au dépôt et à la diffusion de documents scientifiques de niveau recherche, publiés ou non, émanant des établissements d'enseignement et de recherche français ou étrangers, des laboratoires publics ou privés. 


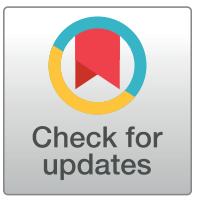

\section{Gopenaccess}

Citation: Benichou T, Pereira B, Mermillod M, Tauveron I, Pfabigan D, Maqdasy S, et al. (2018) Heart rate variability in type 2 diabetes mellitus: $A$ systematic review and meta-analysis. PLoS ONE 13(4): e0195166. https://doi.org/10.1371/journal. pone.0195166

Editor: Rayaz A Malik, Weill Cornell MedicineQatar, QATAR

Received: January 21, 2018

Accepted: March 16, 2018

Published: April 2, 2018

Copyright: $\odot 2018$ Benichou et al. This is an open access article distributed under the terms of the Creative Commons Attribution License, which permits unrestricted use, distribution, and reproduction in any medium, provided the original author and source are credited.

Data Availability Statement: All relevant data are within the paper and its Supporting Information files.

Funding: This study was funded by the University Hospital of Clermont-Ferrand, France.

Competing interests: The authors have declared that no competing interests exist.
RESEARCH ARTICLE

\section{Heart rate variability in type 2 diabetes mellitus: A systematic review and meta- analysis}

\author{
Thomas Benichou ${ }^{1}$, Bruno Pereira ${ }^{2}$, Martial Mermillod ${ }^{3,4}$, Igor Tauveron ${ }^{5}$, \\ Daniela Pfabigan ${ }^{6}$, Salwan Maqdasy ${ }^{5}$, Frédéric Dutheil ${ }^{7,8 *}$
}

1 University Hospital of Clermont-Ferrand, CHU Clermont-Ferrand, Endocrinology, Clermont-Ferrand, France, 2 University Hospital of Clermont-Ferrand, CHU Clermont-Ferrand, Clinical Research Direction, Clermont-Ferrand, France, 3 Univ. Grenoble Alpes, LPNC \& CNRS, LPNC, Grenoble, France, 4 Institut Universitaire de France, Paris, France, 5 Université Clermont Auvergne, CNRS, GReD, Inserm, University Hospital of Clermont-Ferrand, CHU Clermont-Ferrand, Endocrinology, Clermont-Ferrand, France, 6 Peking University, Culture and Social Cognitive Neuroscience Laboratory, School of Psychological and Cognitive Sciences, Beijing, China, 7 Université Clermont Auvergne, CNRS, LaPSCo, Physiological and Psychosocial Stress, University Hospital of Clermont-Ferrand, CHU Clermont-Ferrand, Preventive and Occupational Medicine, WittyFit, Clermont-Ferrand, France, 8 Australian Catholic University, Faculty of Health, School of Exercise Science, Melbourne, Victoria, Australia

*frederic.dutheil@uca.fr

\section{Abstract}

\section{Background}

Cardiac autonomic neuropathy in type 2 dibetes mellitus (T2DM) patients is frequent and associated with high cardiovascular mortality. Heart rate variability (HRV) is the gold standard to measure cardiac autonomic neuropathy. We aimed to conduct a systematic review and meta-analysis to evaluate the impact of T2DM on HRV parameters.

\section{Methods}

The PubMed, Cochrane Library, Embase and Science Direct databases were searched on $1^{\text {st }}$ October 2017 using the keywords "diabetes" AND ("heart rate variability" OR "HRV"). Included articles had to report HRV parameters in T2DM patients and healthy controls measured during 24 hours with a Holter-electrocardiogram. Measurements of HRV retieved were: RR-intervals (or Normal to Normal intervals-NN), standard deviation of RR intervals (SDNN), percetange of adjacent NN intervals differing by more than 50 milliseconds (pNN50), square root of the mean squared difference of successive RR intervals (RMSSD), total power, Low Frequency (LF), High Frequency (HF) and LF/HF ratio, as per Task Force recommendations.

\section{Results}

We included twenty-five case-control studies with 2,932 patients: 1,356 with T2DM and 1,576 healthy controls. T2DM patients had significantly $(\mathrm{P}<0.01)$ lower RR-intervals (effect size $=-0.61 ; 95 \% \mathrm{Cl}-1.21$ to -0.01$)$, lower SDNN $(-0.65 ;-0.83$ to -0.47$)$, lower RMSSD $(-0.92 ;-1.37$ to -0.47$)$, lower pNN50 (-0.46; -0.84 to -0.09$)$, lower total power $(-1.52$; 
-2.13 to -0.91$)$, lower LF $(-1.08 ;-1.46$ to -0.69$])$, and lower HF $(-0.79 ;-1.09$ to -0.50$)$. $\mathrm{LF} / \mathrm{HF}$ did not differ between groups. Levels of blood glucose and $\mathrm{HbA1c}$ were associated with several HRV parameters, as well as Time from diagnosis of T2DM

\section{Conclusions}

T2DM was associated with an overall decrease in the HRV of T2DM patients. Both sympathetic and parasympathetic activity were decreased, which can be explained by the deleterious effects of altered glucose metabolism on HRV, leading to cardiac autonomic neuropathy.

\section{Introduction}

Type 2 diabetes mellitus (T2DM) is a public health concern [1]. T2DM is increasingly frequent in the world in association with the increase of sedentary behaviours, unhealthy diet, obesity and metabolic syndrome [2-6]. The number of people with T2DM is predicted to double within the next three decades [1]. Besides macrovascular [7-10] and microvascular complications [11-13], the leading cause of death in T2DM is cardiovascular mortality [9]. Cardiovascular mortality has been related to the cardiac autonomic neuropathy frequently associated with T2DM $[1,5,14]$.

Screening for cardiac autonomic neuropathy has been recommended at the diagnosis of $\mathrm{T} 2 \mathrm{DM}$, particularly in patients with a history of poor glycaemic control, macro/micro vascular complications, and increased cardiovascular risk [15]. Despite standard cardiovascular reflex tests still belong to the gold standard for the assessment of cardiovascular autonomic neuropathy [16], one of the easiest and most reliable ways to assess cardiac autonomic neuropathy is through the measurement of heart rate variability (HRV). HRV is the variation between two consecutive beats: the higher the variation, the higher the parasympathetic activity. A high HRV reflects the fact that an individual can constantly adapt to micro-environmental changes [17]. Therefore, low HRV is a marker of cardiovascular risk [18]. Conveniently, the measurement of HRV is non-intrusive and pain-free [19]. Although the evaluation of HRV in T2DM has been assessed in several studies, conflicting results have been reported [20-22]. Moreover, there is no consensus on the decreased levels of HRV parameters in T2DM. Furthermore, despite HRV being linked with the severity of T2DM [23], no studies have comprehensively assessed the role of the most common variables, such as age [24], gender [25], blood glucose control [26], or medications treating for T2DM, on HRV parameters [27,28].

Therefore, we aimed to conduct a systematic review and meta-analysis on the impact of T2DM on HRV parameters. A secondary aim was to identify the most frequently reported explanatory variables.

\section{Methods}

\section{Literature search}

We reviewed all studies measuring HRV in T2DM patients and healthy controls. Animal studies were excluded. Between October $30^{\text {th }} 2015$ and October $1^{\text {st }} 2017$, the main articles databases (PubMed, Cochrane Library, Science Direct and Embase) were searched with the following keywords: "diabetes" AND ("heart rate variability" OR "HRV"). All articles compatible with our inclusions criteria were included, independently of article language and years of 


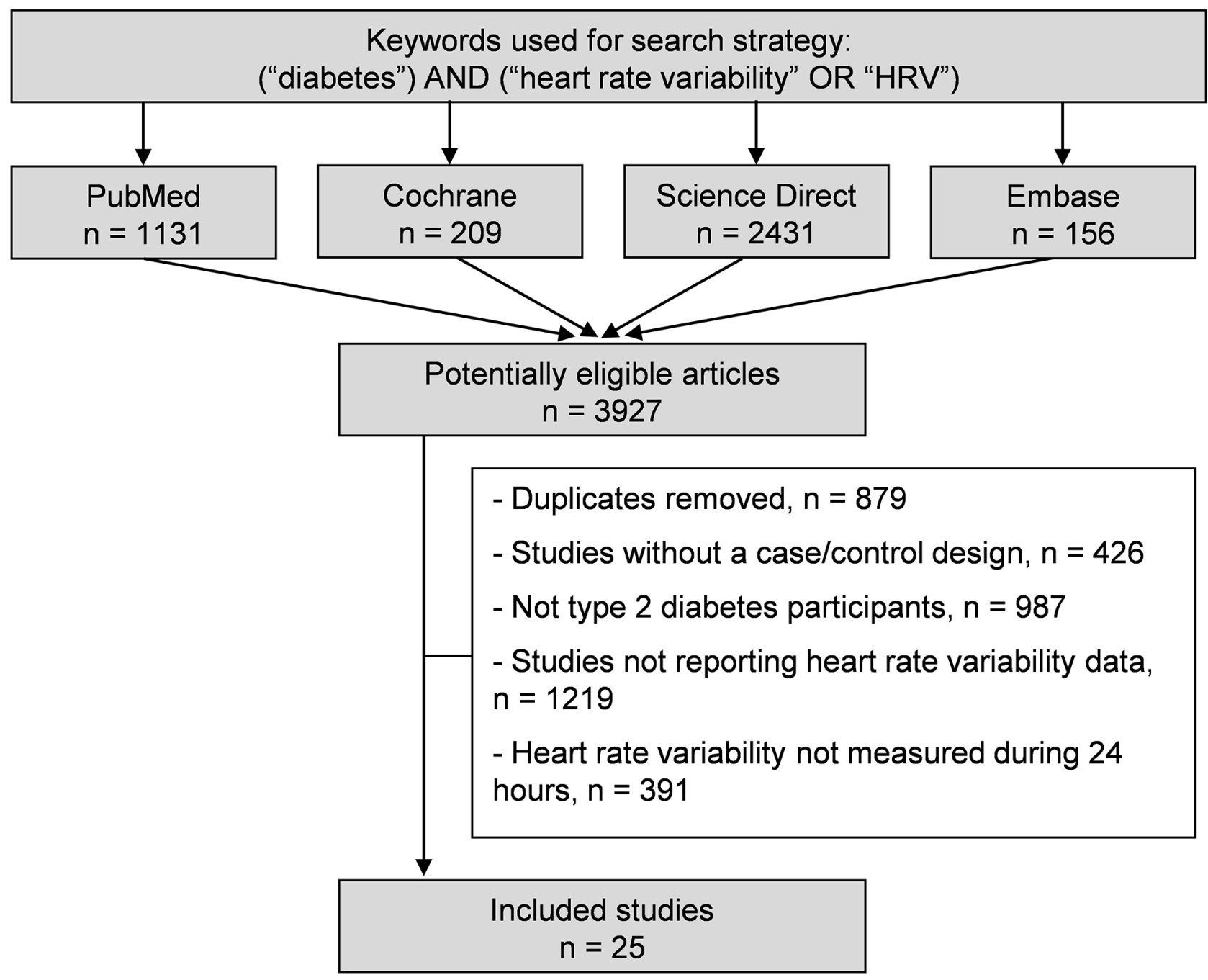

Fig 1. Search strategy.

https://doi.org/10.1371/journal.pone.0195166.g001

publication. To be included, case-control studies had to describe our main primary outcome, which was the measurement of HRV parameters in T2DM patients and healthy controls. We limited included studies to those reporting 24-hour measurements of HRV with Holter-electrocardiogram, following Task Force recommendations [29]. We imposed no limitation on the regional origin or the nature of the control group. Studies needed to be primary research. In addition, reference lists of all publications meeting the inclusion criteria were manually searched to identify any further studies that were not found with the electronic search. Ancestry searches were also completed on previous reviews to locate other potentially eligible primary studies. The search strategy is presented in Fig 1 and in S1 Appendix. One author (Thomas Benichou) conducted the literature searches, collated the articles, and extracted the data. Two authors (Thomas Benichou and Frédéric Dutheil) reviewed the abstracts independently and checked if article could be included in our metanalaysis according to inclusion critera. When consensus on suitability was not reached, a third author (Bruno Pereira) reviewed the debated articles. Then, all authors reviewed the eligible articles. 


\section{Quality of the assessment}

Despite not designed for quantifying the integrity of studies [30], the "STrengthening the Reporting of OBservational studies in Epidemiology" (STROBE) was used for checking the reporting quality of cohorts studies [31]. The STROBE Statement is a checklist of 22 items related to the title, abstract, introduction, methods, results and discussion sections of articles. Cohort, case control, and cross-sectional studies shared 18 items. Four items are specific to each of the three study designs. Six of the 22 items are splitted into several sub-items. We attributed one point per item or sub-item fulfilling the criteria. We calculated a percentage on a maximal score achievable of 32. The Consolidated Standards of Reporting Trials (CONSORT) for checking the reporting quality of randomized trials [32]. Similarly, the 25 items (with 11 of them splitted into several sub-items) from the CONSORT criteria could achieve a maximum score of 37 , then converted into percentage.

\section{HRV parameters}

We included HRV parameters derived from a 24-hour Holter electrocardiogram following task force recommendations [29]. In the time domain, we analysed RR intervals, standard deviations of RR intervals (SDNN), the square root of the mean squared difference of successive RR intervals (RMSSD), and the percentage of adjacent $\mathrm{NN}$ intervals differing by more than 50 milliseconds (pNN50). The RMSSD and pNN50 are associated with high-frequency power (HF) and hence parasympathetic activity, whereas SDNN is correlated with low-frequency power (LF) [33]. In the spectral domain, we analysed LF $(0.04-0.15 \mathrm{~Hz})$, an index of both sympathetic and parasympathetic activity, and $\mathrm{HF}(0.15-0.4 \mathrm{~Hz})$, representing the most efferent vagal (parasympathetic) activity to the sinus node. Very low frequency (VLF; 0.003$0.04 \mathrm{~Hz}$ ) partially reflects thermoregulatory mechanisms, fluctuation in activity of the reninangiotensin system, and the function of peripheral chemoreceptors. The LF/HF ratio, i.e. the sympathovagal balance, was also calculated.

\section{Statistical considerations}

We conducted meta-analyses on the HRV parameters in T2DM patients and healthy controls. $P$ values less than 0.05 were considered statistically significant. For the statistical analysis, we used both Comprehensive Meta-analysis software (version 2, Biostat Corporation) [34-37] and Stata software (version 13, StataCorp, College Station, US) [34-36,38,39]. Main characteristics were synthetized for each study population and reported as the mean \pm SD (standarddeviation) for continuous variables and the number (\%) for categorical variables.

We evaluated heterogeneity in the study results by examining forest plots, confidence intervals (CI) and $\mathrm{I}^{2}$ statistic. Formal tests for homogeneity based on the $\mathrm{I}^{2}$ statistic are the most common metric for measuring the magnitude of between-study heterogeneity and are easily interpretable. $\mathrm{I}^{2}$ values range from 0 to $100 \%$, and are considered low for $<25 \%$, modest for $25-50 \%$, and high for $>50 \%$. We assume heterogeneity for a $\mathrm{p}$-value of the $\mathrm{I}^{2}$ test $<0.05$. For example, a significant heterogeneity could be linked to the characteristics of the studies, such as those of the participants (age, sex, etc.), the time from T2DM diagnosis, the glycaemia or the HbAlc levels. We conducted random effects meta-analyses (DerSimonian and Laird approach) when data could be pooled [40]. To describe our results, we calculated the effect size (ES, standardised mean differences-SMD) [41] of each HRV parameter for each dependent variable. An ES is a unitless measure of the levels of the HRV data. The ES is centered at zero if the HRV data in T2DM patients are not different from those in healthy controls. A positive ES denoted higher levels of the tested HRV parameter in T2DM patients compared with heathy controls. An ES of 0.8 reflects a large effect, 0.5 a moderate effect, and 0.2 a small effect. We 
searched for potential publication bias using funnel plots of these meta-analyses. We verified the strength of our results by conducting further meta-analyses after exclusion of studies that were not evenly distributed around the base of the funnel.

When possible (sufficient sample size), meta-regressions were proposed to study the relationship between each HRV parameter (RR intervals, RMSSD, pNN50, SDNN, total power, LF, HF, LF/HF) and clinically relevant parameters such as gender, age, fasting blood glucose, and glycated haemoglobin (HbAlc). Results were expressed as regression coefficients and 95\% CI.

\section{Results}

An initial search produced a possible 3927 articles (Fig 1). Removal of duplicates and use of the selection criteria reduced the number of articles reporting the evaluation of HRV on 24-hour recording in T2DM to 25 articles [42-66]. All included articles were written in English except one study which was written in Hungarian [55].

\section{Quality of articles}

The assessment of the quality of the twenty-five studies that were included was performed using the STROBE and CONSORT criteria. Results varied from 50\% [59] to 71\% [56] for the observational studies (STROBE), with a mean score of 59\%. Results varied from 52\% [59] to $67 \%$ [57] for the randomized trials (CONSORT), with a mean score of 59.5\%. Overall, the studies performed best in the methods section and worst in the discussion section.

\section{Objectives of included articles}

All included articles aimed to compare HRV between T2DM patients and controls without T2DM [42-66]. Both the T2DM patients and the controls had cardiovascular diseases in five studies $[42,48,51,56,60]$ and renal disease in three studies [43,46,52]. Five studies assessed the influence of high blood pressure on HRV in T2DM compared with healthy controls $[49,50,54,55,58]$. Other studies compared HRV between T2DM patients and controls based on blood catecholamine levels [44], circadian autonomic rhythm in insulinoresistant subjects [45], in cases of bowel preparation [63], metabolic syndrome [47], circadian rhythm in relation to blood adiponectin [62], or dimethylarginine levels [61], hypoglycaemic episodes [59], in acromegalic patients [53], and inhalation of ultrafine particles [57].

\section{Inclusions and exclusions criteria}

For T2DM patients: Inclusion criteria were: aged over 18 [43,48], and under 65 [51], 73 [53], or 75 years old [46,52], treated with oral antidiabetic agents [47], with normal [42,44,45] or high blood pressure [49,61]. The main exclusion criteria were: pregnancy [43,62], neurological disease [43]. Body Mass Index (BMI) over $25 \mathrm{~kg} / \mathrm{m}^{2}$ [42,44,45], or $35 \mathrm{~kg} / \mathrm{m}^{2}$ [58,62], chronic heart $[42,44,45,50-52,57,58,60-63]$, liver [56,60,63], or renal [42,42,45,56,58,60-63] failure, insulin treatment [47,51], uncontrolled T2DM [47], thyroid disorder [50,52], or treatment that can influence HRV parameters [44-46,52,54,57-60].

For controls: All studies included controls without T2DM [42-66]. In each individual study, exclusion criteria were the same as for T2DM, i.e. pregnancy [43,62], neurological disease [43], BMI over $25 \mathrm{~kg} / \mathrm{m}^{2}[42,44,45]$ or $35 \mathrm{~kg} / \mathrm{m}^{2}[58,62]$, chronic heart $[42,44,45,50-52,57,58,60-$ 63], liver [56,60,63], or renal $[42,42,45,56,58,60-63]$ failure, thyroid disorder [50,52], or treatment that can influence HRV parameters [44-46,52,54,57-60]. Controls had high blood pressure in five studies $[49,56,58,60,62]$, and were on dialysis in three studies $[43,46,52]$. 
Healthy controls were paired with T2DM patients based on age $[41,43,44,46,47,49,50,52$, $53,58,59,62]$, gender [42,44,45,47,51,59,60,63], body weight [50,59], BMI [44], and blood pressure [60].

\section{Population}

Sample size. Population sizes ranged from 12 [59], to 457 [56]. We included 2,932 patients in total: 1,356 with T2DM and 1,576 healthy controls.

Gender. The proportion of men varied from 35\% [45] to 100\% [50] in T2DM patients, with a mean of $52.2 \%$, and from $28 \%$ [54] to $100 \%$ [60] in the control group, with a mean of $54.5 \%$. One study did not specify the proportion of men with T2DM [53], while 3 studies did not specify it for the controls $[53,57,59]$.

Age. The mean age of T2DM patients was 58.1 \pm 6.5 years, ranging from 45.9 [57] to 67.9 [52], and 55.9 \pm 7.6 years in the controls, ranging from 28.5 [57] to 65.7 [52]. Age was not reported for T2DM patients in two studies [50,53], and for the controls in three studies $[50,53,59]$.

T2DM duration: the mean time from T2DM diagnosis was $7.8 \pm 4.4$ years ranging from 3.0 $[47,54]$ to 11.2 [51] years. T2DM duration was not reported in five studies $[45,48,50,52,53]$.

Metabolic control. The mean $\mathrm{HbAlc}$ in T2DM patients was $7.6 \pm 0.8 \%$, ranging from 6.5 [59] to $9.3 \%$ [58,62], and 5.2 $\pm 0.5 \%$ in the controls, ranging from 4.1 [42] to 5.8\% [58,62]. Eight studies did not report HbA1c in the T2DM patients $[43,45,46,48,50,53,61,64]$ and nine studies did not specify for the controls $[43,45-48,50,53,61,64]$. Blood glucose levels were reported in 14 studies $[42,44,46,47,51,52,55,58,60-63,65,66]$ with a mean blood glucose level of $147 \pm 16 \mathrm{mg} /$

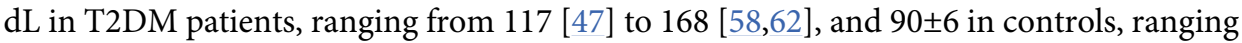
from 75 [46] to 95 [55]. Blood insulin levels were reported in one study [44]. No studies reported HOMA-IR. No studies reported neuropathy scores.

Body weight. Weight was reported in three studies [43,46,49], and waist circumference in two studies [47,52]. The mean BMI in T2DM patients was $27.2 \pm 3.5 \mathrm{~kg} / \mathrm{m}^{2}$, ranging from 22.3 [44] to 33.7 [58,62], and 24.8 \pm 2.1 in controls, ranging from 21.8 [61] to 29.7 [54]. BMI was not reported in 11 studies in T2DM patients [42,43,46,48,50-53,55,57,64], and in 12 studies in controls [42,43,46,48,50-53,55,57,59,64].

Blood pressure. Mean systolic blood pressure in T2DM patients was $138.6 \pm 11.0 \mathrm{mmHg}$, ranging from 121.2 [44] to 156.0 [49], and $137.7 \pm 11.6 \mathrm{mmHg}$ in controls, ranging from 120.2 [45] to 153.0 [49]. Mean diastolic blood pressure in T2DM patients was $80.8 \pm 4.3 \mathrm{mmHg}$, ranging from 69.9 [50] to 90.1 [49] and $82.2 \pm 5.4 \mathrm{mmHg}$ in controls, ranging from 71.9 [50] to $92.9[58,62]$. Six studies did not report blood pressure in either T2DM patients or controls $[42,48,52,53,57,64]$.

Blood lipid levels. Total cholesterol was reported in nine studies $[42,47,51,54,55,61,62,65$, 66]. HDL and LDL cholesterol in six studies [47,55,60-62,65] and triglycerides in 12 studies $[42,47,51,55,56,58-62,65,66]$.

Other characteristics. Smoking was reported in six studies [44,54-57,60], and alcohol in one study [44]. Marital status was never reported. Insufficient data precluded further analyses of those parameters.

\section{Study designs}

All studies described a prospective cohort design, except one study which was a randomly controlled prospective study [57]. 


\section{HRV measurements and analysis}

All included studies measured HRV over 24 consecutive hours using a Holter-electrocardiogram. HRV measurement recording was ambulatory with normal daily activity in most of the studies [42-45,47-51,53,54,56-58,60,62,64-66], ambulatory in non-dialysed patients and during hospitalization for dialysed patients in two studies [46,52], and exclusively during hospitalization in one study [59]. Three studies did not report the conditions of the measurements $[55,61,63]$.

Nearly all studies had a distinct Holter monitoring system: Del Mar Reynolds Medical [61], CardioSmart Institutional CS 550 software [58], Holter cardio Light digital [62], Zymed Medical Instruments [48], Mortara Instruments [53,57], TM 2421 \& 2425 systems [56], Holter AD35 TOP [46], Marquette Electronics [43], Meditech Cardiotens [54,55], Fukuda System [44], Spiderview Holter [52], Cardioscan DMS300-4 Model [63], Holter Digital Recorder AsPEKT [42,51], ArguSys Holter Monitor [47], CardioDay GETEMED [59]. Schiller Microvit MT-101 [65,66], and A\&D System [49]. Four studies did not report the monitoring system $[45,50,60,64]$, Fifteen studies explicitly mentioned that they followed task force recommandations $[42,44,45,47,48,51-56,58,59,61,62]$. Premature atrial and ventricular beats were automatically discarded and visually checked.

\section{Meta-analyses of HRV values in T2DM}

We noted strong evidence that T2DM patients had significantly lower $R R$ intervals (effect size $=-0.61 ; 95 \% \mathrm{CI}-1.21$ to $-0.01, \mathrm{P}=0.01 ; \mathrm{I}^{2}=91.6 \%$ ) (Fig 2) $[45,46,48,52,54,57]$, lower SDNN (effect size $=-0.65 ; 95 \% \mathrm{CI}-0.83$ to $-0.47 ; \mathrm{P}<0.001 ; \mathrm{I}^{2}=65.1 \%$ ) (Fig 3$)[42,45,47,48$, $50-53,55,57,58,60-66]$, lower $R M S S D$ (effect size $=-0.92$; 95\%CI -1.37 to -0.47 ); $\mathrm{P}<0.001$; $\mathrm{I}^{2}=94.0 \%$ ) (Fig 4) $[45,47,48,50,52,53,56-58,60-66]$, lower $p N N 50$ (effect size $=-0.46 ; 95 \% \mathrm{CI}-$ 0.84 to $-0.09 ; \mathrm{P}=0.001 ; \mathrm{I}^{2}=85.5 \%$ ) (Fig 5) $[42,48,50-53,58,60,62,65,66]$, lower total power (effect size $=-1.52 ; 95 \% \mathrm{CI}-2.13$ to $\left.-0.91 ; \mathrm{P}<0.001 ; \mathrm{I}^{2}=93.5 \%\right)$ (Fig 6) $[45-48,50,52,54,65$, 66], lower $L F$ (effect size $=-1.08 ; 95 \% \mathrm{CI}-1.46$ to $\left.-0.69 ; \mathrm{p}<0.001 ; \mathrm{I}^{2}=91.3 \%\right)$ (Fig 7 ) $[42,45,47-54,57,63,65,66]$, and lower $H F$ (effect size $=-0.79 ; 95 \%$ CI -1.09 to $-0.50 ; \mathrm{P}<0.001$; $\mathrm{I}^{2}=85.6 \%$ ) (Fig 8) $[42,44,45,47-54,57,63,65,66] . L F / H F$ did not differ between groups (effect size $=0.02 ; 95 \% \mathrm{CI}-0.38$ to $0.43 ; \mathrm{P}=0.914 ; \mathrm{I}^{2}=90.1 \%$ ) (Fig 9) $[42,44,46-48,50-54,57,65,66]$. Heterogeneity was significant $(\mathrm{P}<0.001)$ for all meta-analyses. Funnel plots of meta-analyses analysing for potential publication bias are presented in the supplementary file. Meta-analyses were reperformed after the exclusion of studies that were not evenly distributed around the base of the funnel and showed similar results.

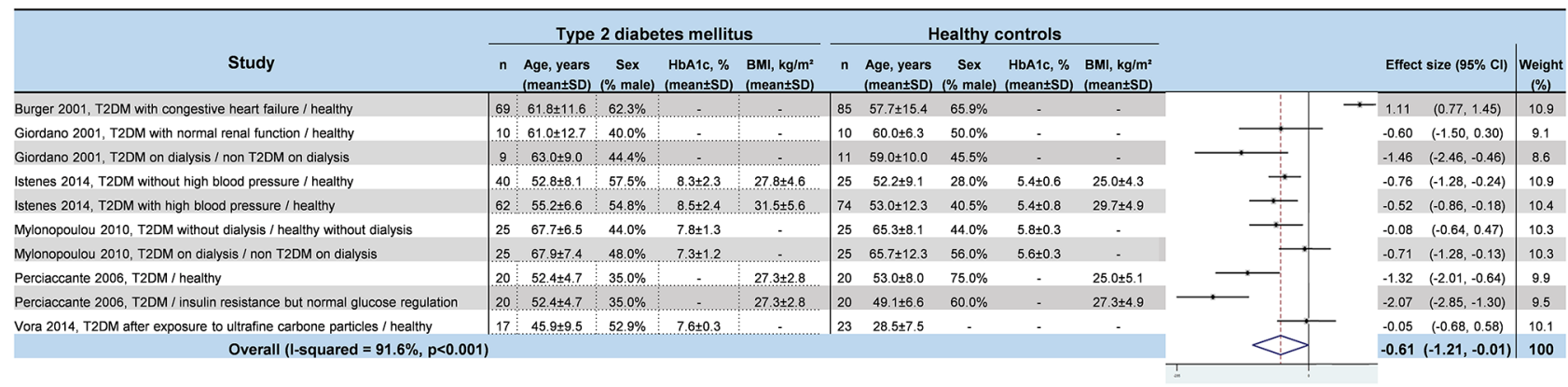

Fig 2. Meta-analysis of RR intervals of type 2 diabetes mellitus patients compared with controls. -: non reported data (missing SD were also non reported). 95\% CI: 95\% confident intervals; BMI: Body Mass Index; RR: RR intervals; T2DM: Type 2 Diabetes Mellitus; SD: Standard Deviation.

https://doi.org/10.1371/journal.pone.0195166.g002 


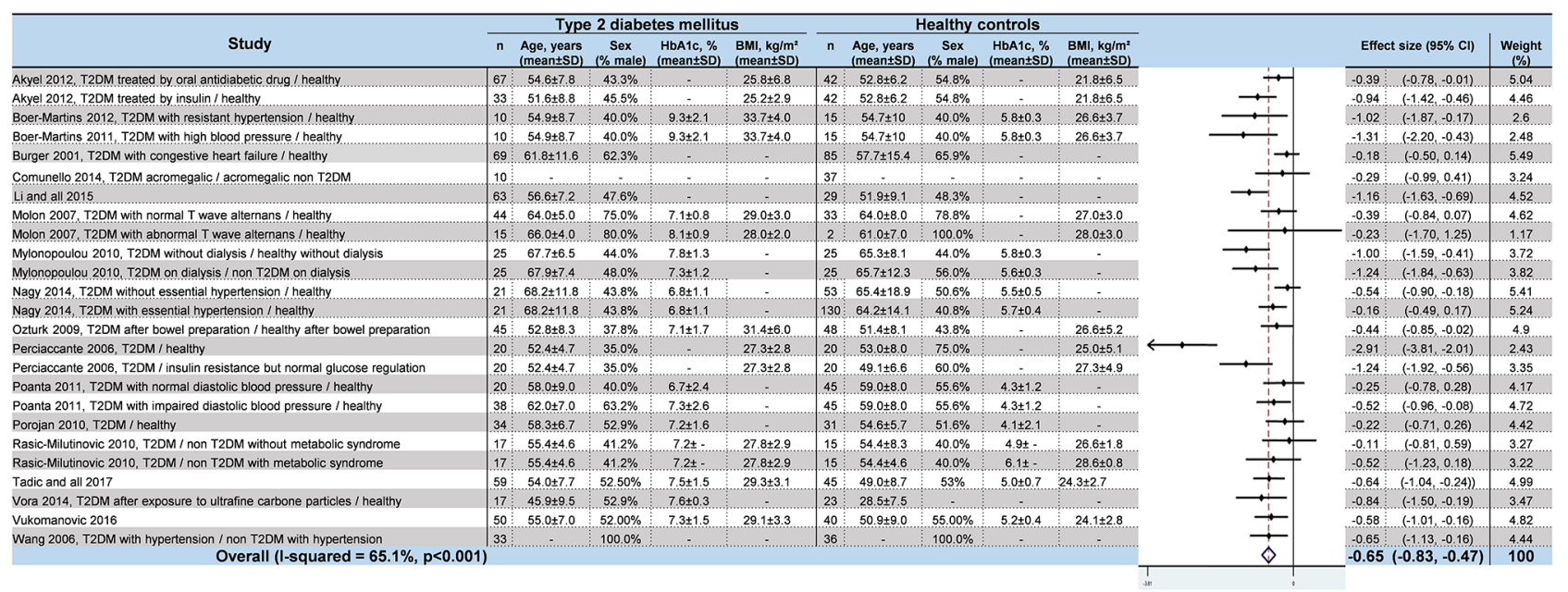

Fig 3. Meta-analysis of SDNN of type 2 diabetes mellitus patients compared with controls. -: non reported data (missing SD were also non reported). 95\% CI: 95\% confident intervals; BMI: Body Mass Index; SDNN: Standard Deviation of RR intervals; T2DM: Type 2 Diabetes Mellitus; SD: Standard Deviation.

https://doi.org/10.1371/journal.pone.0195166.g003

\section{Meta-regressions}

Age and male gender were associated with both a decrease in LF (coefficient $=-0.7 ; 95 \% \mathrm{CI}-1$ to $-0.4, \mathrm{P}<0.001$; and coefficient $=-0.3 ; 95 \% \mathrm{CI}-0.4$ to $-0.1, \mathrm{P}=0.006$, respectively) and a decrease in $\mathrm{HF}$ (coefficient $=-0.6 ; 95 \% \mathrm{CI}-0.9$ to $-0.3, \mathrm{P}<0.001$; and coefficient $=-0.3 ; 95 \%$ CI -0.4 to $-0.1, \mathrm{P}=0.006)$. Similarly, both blood glucose levels and total cholesterol were associated with both an increase in LF (coefficient $=112 ; 95 \% \mathrm{CI} 31-193, \mathrm{P}=0.015$; coefficient $=1133$; $95 \% \mathrm{CI} 408-1858, \mathrm{P}=0.01$; respectively) and in $\mathrm{HF}$ (coefficient $=34$; 95\% CI 6-63, $\mathrm{P}=0.024$; coefficient $=490 ; 95 \% \mathrm{CI} 100-879, \mathrm{P}=0.023)$. Blood glucose levels were also significantly associated with an increase in RMSSD (coefficient $=4.2$; 95\%CI 1.7-6.6, $\mathrm{P}=0.003$ ) and with an increase in SDNN (coefficient $=24 ; 95 \% \mathrm{CI} 17-30, \mathrm{P}<0.001$ ). Higher levels of $\mathrm{HbAlc}$ were

\begin{tabular}{|c|c|c|c|c|c|c|c|c|c|c|c|c|c|}
\hline \multirow[b]{2}{*}{ Study } & \multicolumn{5}{|c|}{ Type 2 diabetes mellitus } & \multicolumn{5}{|c|}{ Healthy controls } & & \multirow[b]{2}{*}{ Effect size $(95 \% \mathrm{Cl})$} & \multirow[b]{2}{*}{$\begin{array}{l}\text { Weight } \\
(\%)\end{array}$} \\
\hline & $n$ & $\begin{array}{l}\text { Age, years } \\
\text { (mean } \pm \text { SD) }\end{array}$ & $\begin{array}{c}\text { Sex } \\
(\% \text { male })\end{array}$ & $\begin{array}{l}\text { HbA1c, \% } \\
\text { (meantSD) }\end{array}$ & $\begin{array}{l}\mathrm{BMI}, \mathrm{kg} / \mathrm{m}^{2} \\
(\mathrm{mean} \pm \mathrm{SD})\end{array}$ & $n$ & $\begin{array}{l}\text { Age, years } \\
\text { (mean } \pm S D)\end{array}$ & $\begin{array}{c}\text { Sex } \\
(\% \text { male })\end{array}$ & $\begin{array}{l}\text { HbA1c, \% } \\
\text { (meantSD) }\end{array}$ & $\begin{array}{l}\mathrm{BMI}, \mathrm{kg} / \mathrm{m}^{2} \\
(\text { (meantSD) }\end{array}$ & & & \\
\hline Akyel 2012. T2DM treated by oral antidiabetic drug / healthy. & 67 & $54.6 \pm 7.8$ & $43.3 \%$ & & $25.8 \pm 6.8$ & 42 & $52.8 \pm 6.2$ & $54.8 \%$ & & $21.8 \pm 6.5$ & & $-1.08 \quad(-1.49,-0.67)$ & 5.08 \\
\hline Akyel 2012, T2DM treated by insulin / healthy & 33 & $51.6 \pm 8.8$ & $45.5 \%$ & - & $25.2 \pm 2.9$ & 42 & $52.8 \pm 6.2$ & $54.8 \%$ & - & $21.8 \pm 6.5$ & $\rightarrow$ & $-1.78 \quad(-2.32,-1.24)$ & 4.93 \\
\hline Boer-Martins 2012, T2DM with resistant hypertension / healthy & 10 & $54.9 \pm 8.7$ & $40.0 \%$ & $9.3 \pm 2.1$ & $33.7 \pm 4$ & 15 & $54.7 \pm 10$ & $40.0 \%$ & $5.8 \pm 0.3$ & $26.6 \pm 3.7$ & & $-1.51 \quad(-2.42,-0.60)$ & 4.37 \\
\hline Burger 2001, T2DM with congestive heart failure / healthy & 69 & $61.8 \pm 11.6$ & $62.3 \%$ & - & - & 85 & $57.7 \pm 15.4$ & $65.9 \%$ & - & - & & $-0.11 \quad(-0.42,0.21)$ & 5.17 \\
\hline Comunello 2014, T2DM acromegalic / acromegalic non T2DM & 10 & - & - & - & - & 37 & - & - & - & - & & $-0.03 \quad(-0.73,0.67)$ & 4.71 \\
\hline Eguchi 2010, T2DM / healthy & 200 & $66.1 \pm 8.9$ & $47.0 \%$ & $7.4 \pm 1.1$ & $24.1 \pm 3.8$ & 257 & $67.5 \pm 8$ & $31.1 \%$ & 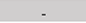 & $23.8 \pm 3.1$ & & $-0.32(-0.51,-0.14)$ & 5.26 \\
\hline Li and all 2015 & 63 & $56.6 \pm 7.2$ & $47.6 \%$ & - & 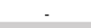 & 29 & $51.9 \pm 9.1$ & $48.3 \%$ & - & - & & $0.35 \quad(-0.1,0.8)$ & 5.05 \\
\hline Molon 2007, T2DM with normal T wave alternans / healthy & 44 & $64.0 \pm 5.0$ & $75.0 \%$ & $7.1 \pm 0.8$ & $29.0 \pm 3.0$ & 33 & $64.0 \pm 9.4$ & $78.8 \%$ & & $27.0 \pm 3.0$ & & $0.56 \quad(0.10,1.02)$ & 5.03 \\
\hline Mylonopoulou 2010, T2DM without dialysis / healthy without dialysis & 25 & $67.7 \pm 6.5$ & $44.0 \%$ & $7.8 \pm 1.3$ & $20.0-2.0$ & 25 & $65.3 \pm 8.1$ & $44.0 \%$ & $5.8 \pm 0.3$ & 20.0 & & $-0.44 \quad(-1.00,0.12)$ & 4.91 \\
\hline Mylonopoulou 2010, T2DM on dialysis / non T2DM on dialysis & 25 & $67.9 \pm 7.4$ & $48.0 \%$ & $7.3 \pm 1.2$ & - & 25 & $65.7 \pm 12.3$ & $56.0 \%$ & $5.6 \pm 0.3$ & - & & $0.03 \quad(-0.52,0.59)$ & 4.9 \\
\hline Ozturk 2009, T2DM after bowel preparation / healthy after bowel preparation & 45 & $52.8 \pm 8.3$ & $37.8 \%$ & $7.1 \pm 1.7$ & $31.4 \pm 6.0$ & 48 & $51.4 \pm 8.1$ & $43.8 \%$ & - & $26.6 \pm 5.2$ & & $-0.42(-0.83,-0.06)$ & 5.08 \\
\hline Perciaccante 2006, T2DM / healthy & 20 & $52.4 \pm 4.7$ & $35.0 \%$ & - & $27.3 \pm 2.8$ & 20 & $53.0 \pm 8.0$ & $75.0 \%$ & - & $25.0 \pm 5.1$ & & $-0.30 \quad(-0.92,0.32)$ & 4.82 \\
\hline Perciaccante 2006, T2DM / insulin resistance but normal glucose regulation & 20 & $52.4 \pm 4.7$ & $35.0 \%$ & - & $27.3 \pm 2.8$ & 20 & $49.1 \pm 6.6$ & $60.0 \%$ & - & $27.3 \pm 4.9$ & & $-0.57 \quad(-1.21,0.06)$ & 4.81 \\
\hline Rasic-Milutinovic 2010, T2DM / non T2DM without metabolic syndrome & 17 & $55.4 \pm 4.6$ & $41.2 \%$ & $7.2 \pm-$ & $27.8 \pm 2.9$ & 15 & $54.4 \pm 8.3$ & $40.0 \%$ & $4.9 \pm-$ & $26.6 \pm 1.8$ & & $-0.13 \quad(-0.82,0.57)$ & 4.72 \\
\hline Rasic-Milutinovic 2010, T2DM / non T2DM with metabolic syndrome & 17 & $55.4 \pm 4.6$ & $41.2 \%$ & $7.2 \pm-$ & $27.8 \pm 2.9$ & 15 & $54.4 \pm 4.6$ & $40.0 \%$ & $6.1 \pm$ - & $28.6 \pm 0.8$ & & $-1.00 \quad(-1.74,-0.26)$ & 4.65 \\
\hline Tadic and all 2017 & 59 & $54.0 \pm 7.7$ & $52.50 \%$ & $7.5 \pm 1.5$ & $29.3 \pm 3.1$ & 45 & $49.0 \pm 8.7$ & $53 \%$ & $5.0 \pm 0.7$ & $24.3 \pm 2.7$ & & $-6.00 \quad(-6.91,-5.09)$ & 4.37 \\
\hline Vora 2014, T2DM after exposure to ultrafine carbone particles / healthy & 17 & $45.9 \pm 9.5$ & $52.9 \%$ & $7.6 \pm 0.3$ & & 23 & $28.5 \pm 7.5$ & & & & & $-0.70(-1.34,-0.05)$ & 4.79 \\
\hline Vukomanovic 2016 & 50 & $55.0 \pm 7.0$ & $52.00 \%$ & $7.3 \pm 1.5$ & $29.1 \pm 3.3$ & 40 & $50.9 \pm 9.0$ & $55.00 \%$ & $5.2 \pm 0.4$ & $24.1 \pm 2.8$ & $\rightarrow$ & $-4.95(-5.79,-4.11)$ & 4.48 \\
\hline $\begin{array}{l}\text { Wang 2006, T2DM with hypertension / non T2DM with hypertension } \\
\text { Overall (I-squared }=94.0 \%,\end{array}$ & 33 & - & $100.0 \%$ & & 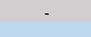 & 36 & 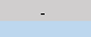 & $100.0 \%$ & & & 世 & $\begin{array}{ll}-0.62 & (-1.10,-0.13) \\
-0.92 & (-1.37,-0.47)\end{array} \mid$ & $\begin{array}{c}5 \\
100\end{array}$ \\
\hline
\end{tabular}

Fig 4. Meta-analysis of RMSSD of type 2 diabetes mellitus patients compared with controls. -: non reported data (missing SD were also non reported). 95\% CI: 95\% confident intervals; BMI: Body Mass Index; RMSSD: square root of mean squared differences of successive RR intervals; T2DM: Type 2 Diabetes Mellitus; SD: Standard Deviation.

https://doi.org/10.1371/journal.pone.0195166.g004 


\begin{tabular}{|c|c|c|c|c|c|c|c|c|c|c|c|c|c|c|}
\hline \multirow[b]{2}{*}{ Study } & \multicolumn{5}{|c|}{ Type 2 diabetes mellitus } & \multicolumn{5}{|c|}{ Healthy controls } & & \multirow{2}{*}{\multicolumn{2}{|c|}{ Effect size $(95 \%$ Cl) }} & \multirow[b]{2}{*}{$\begin{array}{c}\text { Weight } \\
(\%)\end{array}$} \\
\hline & $\mathrm{n}$ & $\begin{array}{l}\text { Age, years } \\
\text { (meantSD) }\end{array}$ & $\begin{array}{c}\text { Sex } \\
\text { (\% male) }\end{array}$ & $\begin{array}{l}\text { HbA1c, \% } \\
\text { (mean } \pm \text { SD) }\end{array}$ & $\begin{array}{l}\mathrm{BMI}, \mathrm{kg} / \mathrm{m}^{2} \\
(\mathrm{mean} \pm \mathrm{SD})\end{array}$ & $n$ & $\begin{array}{l}\text { Age, years } \\
\text { (meantSD) }\end{array}$ & $\begin{array}{c}\text { Sex } \\
(\% \text { male })\end{array}$ & $\begin{array}{l}\text { HbA1c, \% } \\
\text { (mean } \pm S D)\end{array}$ & $\begin{array}{l}\mathrm{BMI}, \mathrm{kg} / \mathrm{m}^{2} \\
(\text { mean } \pm \mathrm{SD})\end{array}$ & & & & \\
\hline Boer-Martins 2012, T2DM with resistant hypertension / healthy & 10 & $54.9 \pm 8.7$ & $40.0 \%$ & $9.3 \pm 2.1$ & $33.7 \pm 4$ & 15 & $54.7 \pm 10$ & $40.0 \%$ & $5.8 \pm 0.3$ & $26.6 \pm 3.7$ & & -0.68 & $(-1.50,0.15)$ & 6.2 \\
\hline Boer-Martins 2011, T2DM with high blood pressure / healthy & 10 & $54.9 \pm 8.7$ & $40.0 \%$ & $9.3 \pm 2.1$ & $33.7 \pm 4$ & 15 & $54.7 \pm 10$ & $40.0 \%$ & $5.8 \pm 0.3$ & $26.6 \pm 3.7$ & & -0.70 & $(-1.53,0.12)$ & 6.19 \\
\hline Burger 2001, T2DM with congestive heart failure / healthy & 69 & $61.8 \pm 11.6$ & $62.3 \%$ & - & - & 85 & $57.7 \pm 15.4$ & $65.9 \%$ & - & - & $\rightarrow$ & -0.95 & $(-1.28,-0.61)$ & 8.24 \\
\hline Comunello 2014 T2DM acromegalic / acromegalic non T2DM & 10 & - & - & - & - & 37 & - & - & - & - & & 0.33 & $(-0.37,1.03)$ & 6.75 \\
\hline Molon 2007, T2DM with normal T wave alternans / healthy & 44 & $64.0 \pm 5.0$ & $75.0 \%$ & $7.1 \pm 0.8$ & $29 \pm 3$ & 33 & $64.0 \pm 8.0$ & $78.8 \%$ & - & $27 \pm 3$ & & 0.53 & $(0.07,0.99)$ & 7.79 \\
\hline Molon 2007 T2DM with abnormal T wave alternans / healthy & 15 & $66.0 \pm 4.0$ & $80.0 \%$ & $8.1 \pm 0.9$ & $28 \pm 2$ & 2 & $61.0 \pm 7.0$ & $100.0 \%$ & - & $28 \pm 3$ & & -0.39 & $(-1.88,1.09)$ & 3.73 \\
\hline Mylonopoulou 2010, T2DM without dialysis / healthy without dialysis & 25 & $67.7 \pm 6.5$ & $44.0 \%$ & $7.8 \pm 1.3$ & - & 25 & $65.3 \pm 8.1$ & $44.0 \%$ & $5.8 \pm 0.3$ & - & & -0.47 & $(-1.03,0.10)$ & 7.38 \\
\hline Mylonopoulou 2010. T2DM on dialysis / non T2DM on dialysis & 25 & $67.9 \pm 7.4$ & $48.0 \%$ & $7.3 \pm 1.2$ & - & 25 & $65.7 \pm 12.3$ & $56.0 \%$ & $5.6 \pm 0.3$ & - & & 0.39 & $(-0.17,0.95)$ & 7.37 \\
\hline Poanta 2011, T2DM with normal diastolic blood pressure / healthy & 20 & $58.0 \pm 9.0$ & $40.0 \%$ & $6.7 \pm 2.4$ & - & 45 & $59.0 \pm 8.0$ & $55.6 \%$ & $4.3 \pm 1.2$ & & & -0.15 & $(-0.68,0.38)$ & 7.52 \\
\hline Porojan 2010, T2DM / healthy & 34 & $58.3 \pm 6.7$ & $52.9 \%$ & $7.2 \pm 1.6$ & - & 31 & $54.6 \pm 5.7$ & $51.6 \%$ & $4.1 \pm 2.1$ & & & -0.15 & $(-0.63,0.34)$ & 7.68 \\
\hline Tadic and all 2017 & 59 & $54.0 \pm 7.7$ & $52.50 \%$ & $7.5 \pm 1.5$ & $29.3 \pm 3.1$ & 45 & $49.0 \pm 8.7$ & $53 \%$ & $5.0 \pm 0.7$ & $24.3 \pm 2.7$ & & -1.75 & $(-2.20,-1.29)$ & 7.8 \\
\hline Vukomanovic 2016 & 50 & $55.0 \pm 7.0$ & $52.00 \%$ & $7.3 \pm 1.5$ & $29.1 \pm 3.3$ & 40 & $50.9 \pm 9.0$ & $55.00 \%$ & $5.2 \pm 0.4$ & $24.1 \pm 2.8$ & & -1.43 & $(-1.90,-0.97)$ & 7.76 \\
\hline $\begin{array}{l}\text { Wang 2006, T2DM with hypertension / non T2DM with hypertension } \\
\text { Overall (I-squared }=85.5 \%, p<0.001)\end{array}$ & 33 & & $100.0 \%$ & . & 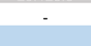 & 36 & 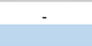 & $100.0 \%$ & & & $\dot{<}$ & -0.55 & $\begin{array}{c}(-1.03,-0.07) \\
(-0.84,-0.09)\end{array}$ & $\begin{array}{c}7.7 \\
100\end{array}$ \\
\hline
\end{tabular}

Fig 5. Meta-analysis of pNN50 of type 2 diabetes mellitus patients compared with controls. -: non reported data (missing SD were also non reported). 95\% CI: 95\% confident intervals; BMI: Body Mass Index; pNN50: percentage of RR intervals with more than 50 ms variation; T2DM: Type 2 Diabetes Mellitus; SD: Standard Deviation.

https://doi.org/10.1371/journal.pone.0195166.g005

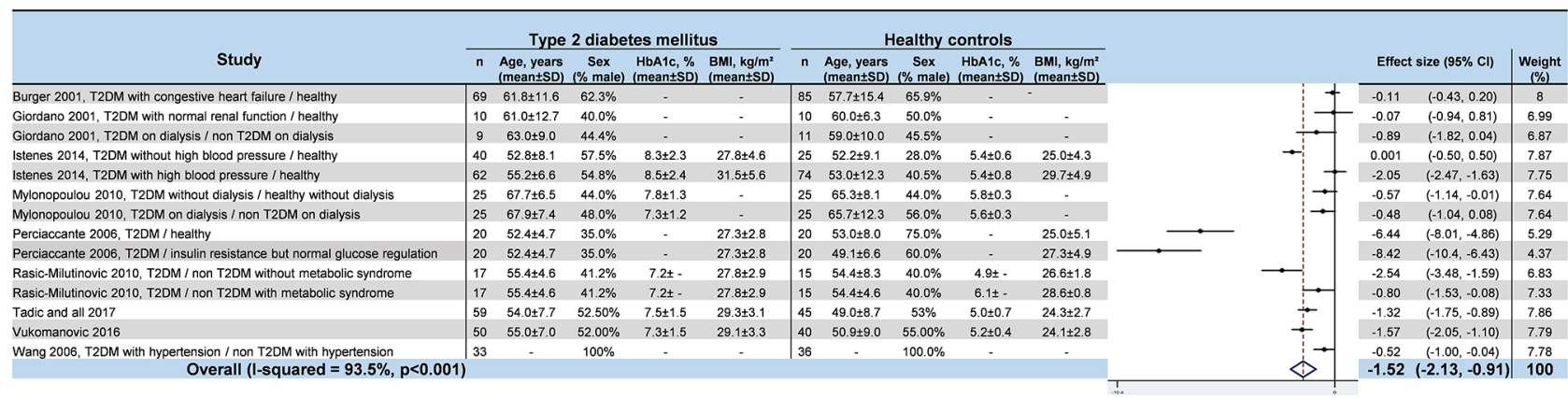

Fig 6. Meta-analysis of total power of type 2 diabetes mellitus patients compared with controls. -: non reported data (missing SD were also non reported). 95\% CI: 95\% confident intervals; BMI: Body Mass Index; TP: Total Power; T2DM: Type 2 Diabetes Mellitus; SD: Standard Deviation.

https://doi.org/10.1371/journal.pone.0195166.g006

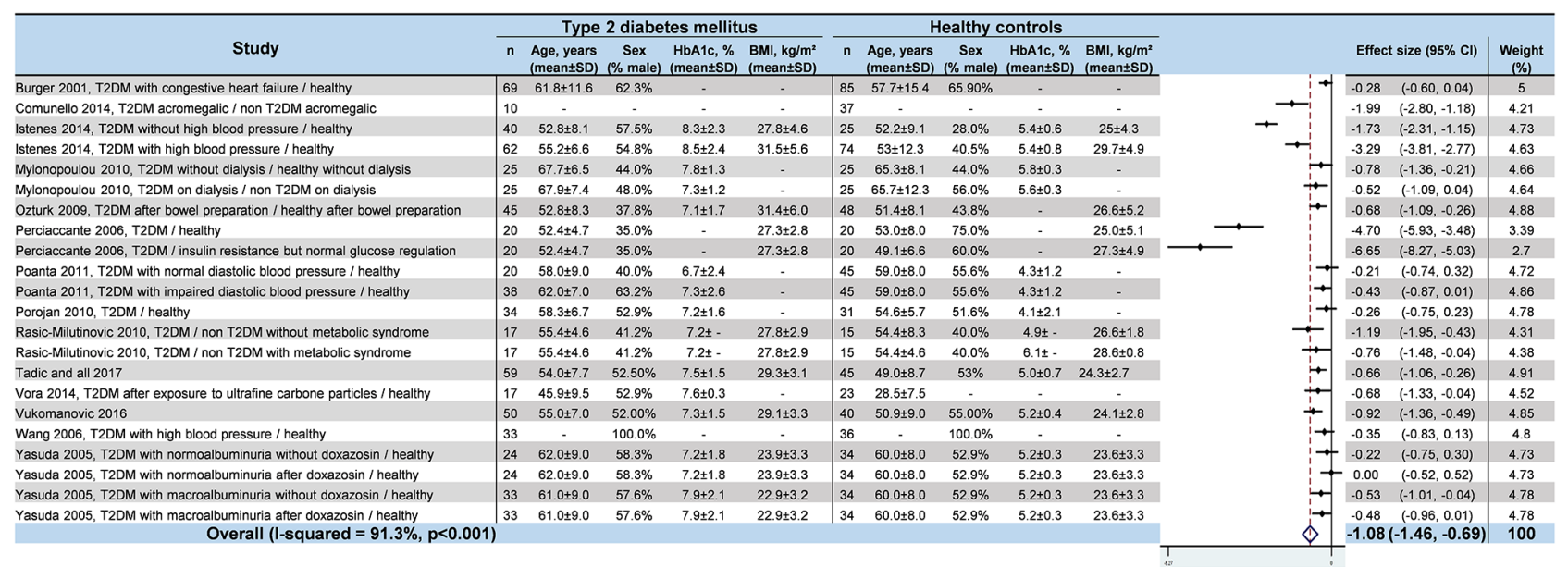

Fig 7. Meta-analysis of the LF of type 2 diabetes mellitus patients compared with controls. -: non reported data (missing SD were also non reported). 95\% CI: 95\% confident intervals; BMI: Body Mass Index; LF: Low Frequency; T2DM: Type 2 Diabetes Mellitus; SD: Standard Deviation.

https://doi.org/10.1371/journal.pone.0195166.g007 


\begin{tabular}{|c|c|c|c|c|c|c|c|c|c|c|c|c|c|}
\hline \multirow[b]{2}{*}{ Study } & \multicolumn{5}{|c|}{ Type 2 diabetes mellitus } & \multicolumn{5}{|c|}{ Healthy controls } & & \multirow[b]{2}{*}{ Effect size $(95 \% \mathrm{Cl})$} & \multirow[b]{2}{*}{$\begin{array}{l}\text { Weight } \\
(\%)\end{array}$} \\
\hline & $n$ & $\begin{array}{l}\text { Age, years } \\
\text { (mean } \pm \text { SD) }\end{array}$ & $\begin{array}{c}\text { Sex } \\
\text { (\% male) }\end{array}$ & $\begin{array}{c}\text { HbA1c, \% } \\
\text { (meantSD) }\end{array}$ & $\begin{array}{r}\mathrm{BMI}, \mathrm{kg} / \mathrm{m}^{2} \\
(\mathrm{mean} \pm \mathrm{SD})\end{array}$ & $n$ & $\begin{array}{l}\text { Age, years } \\
\text { (mean } \pm S D)\end{array}$ & $\begin{array}{c}\text { Sex } \\
\text { (\% male) }\end{array}$ & $\begin{array}{r}\text { HbA1c, \% } \\
\text { (meantSD) }\end{array}$ & $\begin{array}{r}\mathrm{BMI}, \mathrm{kg} / \mathrm{m}^{2} \\
\text { (meantSD) }\end{array}$ & & & \\
\hline Burger 2001, T2DM with congestive heart failure / healthy & 69 & $61.8 \pm 11.6$ & $62.3 \%$ & & & 85 & $57.7 \pm 15.4$ & $65.9 \%$ & & & & $-0.06 \quad(-0.37,0.26)$ & 5.04 \\
\hline Comunello 2014, T2DM acromegalic / non T2DM acromegalic & 10 & & & - & - & 37 & - & - & & - & & $-0.09 \quad(-0.79,0.61)$ & 4.11 \\
\hline Istenes 2014, T2DM without high blood pressure / healthy & 40 & $52.8 \pm 8.1$ & $57.5 \%$ & $8.3 \pm 2.3$ & $27.8 \pm 4.6$ & 25 & $52.2 \pm 9.1$ & $28.0 \%$ & $5.4 \pm 0.6$ & $25.0 \pm 4.3$ & & $-0.35 \quad(-0.9,0.16)$ & 4.9 \\
\hline Istenes 2014, T2DM with high blood pressure / healthy & 62 & $55.2 \pm 6.6$ & $54.8 \%$ & $8.5 \pm 2.4$ & $31.5 \pm 5.6$ & 74 & $53 \pm 12.3$ & $40.5 \%$ & $5.4 \pm 0.8$ & $29.7 \pm 4.9$ & & $-1.61 \quad(-2.00,-1.23)$ & 4.63 \\
\hline Kondo 2002, T2DM / healthy & 10 & $57.6 \pm 8.9$ & $50.0 \%$ & $9.3 \pm 2.5$ & $22.3 \pm 1.3$ & 8 & $53.9 \pm 15$ & $62.5 \%$ & $5.0 \pm 0.3$ & $22.4 \pm 0.6$ & & $-2.04 \quad(-3.21,-0.87)$ & 2.89 \\
\hline Mylonopoulou 2010, T2DM without dialysis / healthy without dialysis & 25 & $67.7 \pm 6.5$ & $44.0 \%$ & $7.8 \pm 1.3$ & 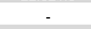 & 25 & $65.3 \pm 8.1$ & $44.0 \%$ & $5.8 \pm 0.3$ & & & $-0.32 \quad(-0.88,0.24)$ & 4.5 \\
\hline Mylonopoulou 2010, T2DM on dialysis / non T2DM on dialysis & 25 & $67.9 \pm 7.4$ & $48.0 \%$ & $7.3 \pm 1.2$ & 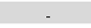 & 25 & $65.7 \pm 12.3$ & $56.0 \%$ & $5.6 \pm 0.3$ & & & $0.10 \quad(-0.45,0.66)$ & 4.49 \\
\hline Ozturk 2009, T2DM after bowel preparation / healthy after bowel preparation & 45 & $52.8 \pm 8.3$ & $37.8 \%$ & $7.1 \pm 1.7$ & $31.4 \pm 6.0$ & 48 & $51.4 \pm 8.1$ & $43.8 \%$ & . & $26.6 \pm 5.2$ & & $-0.51 \quad(-0.92,-0.10)$ & 4.84 \\
\hline Perciaccante 2006, T2DM / healthy & 20 & $52.4 \pm 4.7$ & $35.0 \%$ & 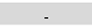 & $27.3 \pm 2.8$ & 20 & $53.0 \pm 8.0$ & $75.0 \%$ & . & $25.0 \pm 5.1$ & 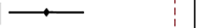 & $-5.92 \quad(-7.39,-4.45)$ & 2.28 \\
\hline Poanta 2011, T2DM with normal diastolic blood pressure / healthy & 20 & $58.0 \pm 9.0$ & $40.0 \%$ & $6.7 \pm 2.4$ & & 45 & $59.0 \pm 8.0$ & $55.6 \%$ & $4.3 \pm 1.2$ & & & $-0.15 \quad(-0.68,0.38)$ & 4.57 \\
\hline Poanta 2011, T2DM with impaired diastolic blood pressure / healthy & 38 & $62.0 \pm 7.0$ & $63.2 \%$ & $7.3 \pm 2.6$ & - & 45 & $59.0 \pm 8.0$ & $55.6 \%$ & $4.3 \pm 1.2$ & - & & $-0.46 \quad(-0.90,-0.02)$ & 4.79 \\
\hline Porojan 2010, T2DM / healthy & 34 & $58.3 \pm 6.7$ & $52.9 \%$ & $7.2 \pm 1.6$ & - & 31 & $54.6 \pm 5.7$ & $51.6 \%$ & $4.1 \pm 2.1$ & 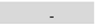 & & $-0.27 \quad(-0.75,0.22)$ & 4.66 \\
\hline Rasic-Milutinovic 2010, T2DM / non T2DM without metabolic syndrome & 17 & $55.4 \pm 4.6$ & $41.2 \%$ & $7.2 \pm$ & $27.8 \pm 2.9$ & 15 & $54.4 \pm 8.3$ & $40.0 \%$ & $4.9 \pm$ & $26.6 \pm 1.8$ & & $-2.34 \quad(-3.25,-1.43)$ & 3.52 \\
\hline Rasic-Milutinovic 2010, T2DM / non T2DM with metabolic syndrome & 17 & $55.4 \pm 4.6$ & $41.2 \%$ & $7.2 \pm$. & $27.8 \pm 2.9$ & 15 & $54.4 \pm 4.6$ & $40.0 \%$ & $6.1 \pm$ - & $28.6 \pm 0.8$ & & $-0.66 \quad(-1.36,0.05)$ & 4.06 \\
\hline Tadic and all 2017 & 59 & $54.0 \pm 7.7$ & $52.50 \%$ & $7.5 \pm 1.5$ & $29.3 \pm 3.1$ & 45 & $49.0 \pm 8.7$ & $53 \%$ & $5.0 \pm 0.7$ & $24.3 \pm 2.7$ & & $-0.66(-1.06,-0.26)$ & 4.88 \\
\hline Vora 2014. T2DM after exposure to ultrafine carbone particles / healthy & 17 & $45.9 \pm 9.5$ & $52.9 \%$ & $7.6 \pm 0.3$ & . & 23 & $28.5 \pm 7.5$ & & & & $+\frac{1}{4}$ & $-0.56 \quad(-1.20,0.08)$ & 4.27 \\
\hline Vukomanovic 2016 & 50 & $55.0 \pm 7.0$ & $52.00 \%$ & $7.3 \pm 1.5$ & $29.1 \pm 3.3$ & 40 & $50.9 \pm 9.0$ & $55.00 \%$ & $5.2 \pm 0.4$ & $24.1 \pm 2.8$ & $\rightarrow$ & $-0.92 \quad(-1.36,-0.49)$ & 4.79 \\
\hline Wang 2006, T2DM with high blood pressure / healthy & 33 & & $100.0 \%$ & & & 36 & 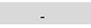 & $100.0 \%$ & & & $\rightarrow$ & $-1.04 \quad(-1.54,-0.54)$ & 4.63 \\
\hline Yasuda 2005, T2DM with normoalbuminuria without doxazosin / healthy & 24 & $62.0 \pm 9.0$ & $58.3 \%$ & $7.2 \pm 1.8$ & $23.9 \pm 3.3$ & 34 & $60.0 \pm 8.0$ & $52.9 \%$ & $5.2 \pm 0.3$ & $23.6 \pm 3.3$ & & $-0.35 \quad(-0.88,0.18)$ & 4.57 \\
\hline Yasuda 2005 , T2DM with normoalbuminuria after doxazosin / healthy & 24 & $62.0 \pm 9.0$ & $58.3 \%$ & $7.2 \pm 1.8$ & $23.9 \pm 3.3$ & 34 & $60.0 \pm 8.0$ & $52.9 \%$ & $5.2 \pm 0.3$ & $23.6 \pm 3.3$ & $\rightarrow$ & $-0.33 \quad(-0.86,0.20)$ & 4.57 \\
\hline Yasuda 2005, T2DM with macroalbuminuria without doxazosin / healthy & 33 & $61.0 \pm 9.0$ & $57.6 \%$ & $7.9 \pm 2.1$ & $22.9 \pm 3.2$ & 34 & $60.0 \pm 8.0$ & $52.9 \%$ & $5.2 \pm 0.3$ & $23.6 \pm 3.3$ & & $-0.37 \quad(-0.85,0.12)$ & 4.68 \\
\hline
\end{tabular}

Fig 8. Meta-analysis of the HF of type 2 diabetes mellitus patients compared with controls. -: non reported data (missing SD were also non reported). 95\% CI: 95\% confident intervals; BMI: Body Mass Index; HF: High Frequency; T2DM: Type 2 Diabetes Mellitus; SD: Standard Deviation.

https://doi.org/10.1371/journal.pone.0195166.g008

associated with shorter $\mathrm{RR}$ intervals (coefficient $=-383 ; 95 \% \mathrm{CI}-756$ to $-8.8, \mathrm{P}=0.046$ ). $H D L$ cholesterol levels were associated with both an increase in SDNN (coefficient $=1251 ; 95 \% \mathrm{CI}$ 292-2210, $\mathrm{P}=0.017$ ) and RMSSD (coefficient $=246$; 95\%CI 186-307, $\mathrm{P}<0.001$ ). Body mass index was associated with both an increase in LF (coefficient $=0.8$; 95\% CI 0.3-1.3, $\mathrm{P}=0.003$ ) and in $\mathrm{HF}$ (coefficient $=0.7 ; 95 \% \mathrm{CI} 0.3-1.1, \mathrm{P}=0.005$ ). An increase in systolic blood pressure was linked with shorter RR intervals (coefficient $=-380 ; 95 \% \mathrm{CI}-703$ to $-53, \mathrm{P}=0.032$ ) and a decrease in $\mathrm{HF}$ (coefficient $=-3.1 ; 95 \% \mathrm{CI}-6.2$ to $-0.02, \mathrm{P}=0.049$ ). Time from diagnosis of T2DM was linked with a higher level of SDNN (coefficient $=10 ; 95 \% \mathrm{CI} 2.1-18, \mathrm{P}=0.018$ ) and a lower level of total power (coefficient $=-1214 ; 95 \% \mathrm{CI}-2129$ to $-299, \mathrm{P}=0.021)($ Fig 10$)$.

\section{Discussion}

The main findings were that T2DM patients exhibited a strong decrease in HRV. Both sympathetic and parasympathetic activity were decreased compared with non-T2DM patients, which can be explained by the deleterious metabolic effects of blood glucose levels on HRV.

\begin{tabular}{|c|c|c|c|c|c|c|c|c|c|c|c|c|c|c|}
\hline \multirow[b]{2}{*}{ Study } & \multicolumn{5}{|c|}{ Type 2 diabetes mellitus } & \multicolumn{5}{|c|}{ Healthy controls } & & & \multirow[b]{2}{*}{ Effect size $(95 \% \mathrm{Cl})$} & \multirow[b]{2}{*}{$\begin{array}{l}\text { Weight } \\
(\%)\end{array}$} \\
\hline & $n$ & $\begin{array}{l}\text { Age, years } \\
\text { (meantSD) }\end{array}$ & $\begin{array}{c}\text { Sex } \\
\text { (\% male) }\end{array}$ & $\begin{array}{l}\text { HbA1c, \% } \\
\text { (meantSD) }\end{array}$ & $\begin{array}{r}\mathrm{BMI}, \mathrm{kg} / \mathrm{m}^{2} \\
\text { (mean } \pm \mathrm{SD} \text { ) }\end{array}$ & $n$ & $\begin{array}{l}\text { Age, years } \\
\text { (mean } \pm S D)\end{array}$ & $\begin{array}{c}\text { Sex } \\
\text { (\% male) }\end{array}$ & $\begin{array}{l}\text { HbA1c, \% } \\
\text { (meantSD) }\end{array}$ & $\begin{array}{l}\mathrm{BMI}, \mathrm{kg} / \mathrm{m}^{2} \\
(\text { mean } \pm \mathrm{SD})\end{array}$ & & & & \\
\hline Burger 2001, T2DM with congestive heart failure / healthy & 69 & $61.8 \pm 11.6$ & $62.3 \%$ & & & 85 & $57.7 \pm 15.4$ & $65.9 \%$ & -- & & $\rightarrow$ & & $-0.45 \quad(-0.78,-0.13)$ & 6.18 \\
\hline Comunello 2014, T2DM acromegalic / non T2DM acromegalic & 10 & . & . & . & - & 37 & - & - & - & - & - & & $-1.88 \quad(-2.68,-1.08)$ & 5.14 \\
\hline Giordano 2001, T2DM with normal renal function / healthy & 10 & $61 \pm 12.7$ & $40.0 \%$ & & 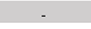 & 10 & $60.0 \pm 6.3$ & $50.0 \%$ & - & - & & & $1.03 \quad(0.09,1.97)$ & 4.78 \\
\hline Istenes 2014, T2DM without high blood pressure / healthy & 40 & $52.8 \pm 8.1$ & $57.5 \%$ & $8.3+2.3$ & $27.8 \pm 4.6$ & 25 & $52.2 \pm 9.1$ & $28.0 \%$ & $5.4 \pm 0.6$ & $25.0 \pm 4.3$ & & + & $0.14 \quad(-0.36,0.64)$ & 6.16 \\
\hline Istenes 2014, T2DM with high blood pressure / healthy & 62 & $55.2 \pm 6.6$ & $54.8 \%$ & $8.5 \pm 2.4$ & $31.5 \pm 5.6$ & 74 & $53 \pm 12.3$ & $40.5 \%$ & $5.4 \pm 0.8$ & $29.7 \pm 4.9$ & & $\leftarrow$ & $0.28 \quad(-0.06,0.61)$ & 5.85 \\
\hline Kondo 2002, T2DM / healthy & 10 & $57.6 \pm 8.9$ & $50.0 \%$ & $9.3 \pm 2.5$ & $22.3 \pm 1.3$ & 8 & $53.9 \pm 15$ & $62.5 \%$ & $5.0 \pm 0.3$ & $22.4 \pm 0.6$ & & & $-1.27 \quad(-2.30,-0.24)$ & 4.54 \\
\hline Mylonopoulou 2010, T2DM without dialysis / healthy without dialysis & 25 & $67.7 \pm 6.5$ & $44.0 \%$ & $7.8 \pm 1.3$ & - & 25 & $65.3 \pm 8.1$ & $44.0 \%$ & $5.8 \pm 0.3$ & - & ـ & & $-0.14 \quad(-0.70,0.42)$ & 5.67 \\
\hline Mylonopoulou 2010, T2DM on dialysis / non T2DM on dialysis & 25 & $67.9 \pm 7.4$ & $48.0 \%$ & $7.3 \pm 1.2$ & - & 25 & $65.7 \pm 12.3$ & $56.0 \%$ & $5.6 \pm 0.3$ & - & & & $-0.89 \quad(-1.48,-0.31)$ & 5.74 \\
\hline Poanta 2011, T2DM with normal diastolic blood pressure / healthy & 20 & $58.0 \pm 9.0$ & $40.0 \%$ & $6.7 \pm 2.4$ & - & 45 & $59.0 \pm 8.0$ & $55.6 \%$ & $4.3 \pm 1.2$ & - & $\rightarrow$ & & $-1.23 \quad(-1.80,-0.66)$ & 5.7 \\
\hline Poanta 2011, T2DM with impaired diastolic blood pressure / healthy & 38 & $62.0 \pm 7.0$ & $63.2 \%$ & $7.3 \pm 2.6$ & - & 45 & $59.0 \pm 8.0$ & $55.6 \%$ & $4.3 \pm 1.2$ & - & $\rightarrow$ & & $-0.59 \quad(-1.03,-0.15)$ & 5.97 \\
\hline Porojan 2010, T2DM / healthy & 34 & $58.3 \pm 6.7$ & $52.9 \%$ & $7.2 \pm 1.6$ & . & 31 & $54.6 \pm 5.7$ & $51.6 \%$ & $4.1 \pm 2.1$ & . & & & $-0.10 \quad(-0.58,0.39)$ & 5.88 \\
\hline Rasic-Milutinovic 2010, T2DM / non T2DM without metabolic syndrome & 17 & $55.4 \pm 4.6$ & $41.2 \%$ & $7.2 \pm-$ & $27.8 \pm 2.9$ & 15 & $54.4 \pm 8.3$ & $40.0 \%$ & $4.9 \pm-$ & $26.6 \pm 1.8$ & & & $0.72 \quad(0.01,1.44)$ & 5.35 \\
\hline Rasic-Milutinovic 2010. T2DM / non T2DM with metabolic syndrome & 17 & $55.4 \pm 4.6$ & $41.2 \%$ & $7.2 \pm$. & $27.8 \pm 2.9$ & 15 & $54.4 \pm 4.6$ & $40.0 \%$ & $6.1 \pm$ - & $28.6 \pm 0.8$ & & - & $0.44 \quad(-0.26,1.14)$ & 5.38 \\
\hline Tadic and all 2017 & 59 & $54.0 \pm 7.7$ & $52.50 \%$ & $7.5 \pm 1.5$ & $29.3 \pm 3.1$ & 45 & $49.0+8.7$ & $53 \%$ & $5.0 \pm 0.7$ & $24.3 \pm 2.7$ & & & $-0.22 \quad(-0.61,0.17)$ & 6.07 \\
\hline Vora 2014, T2DM after exposure to ultrafine carbone particles / healthy & 17 & $45.9 \pm 9.5$ & $52.9 \%$ & $7.6 \pm 0.3$ & & 23 & $28.5 \pm 7.5$ & - & - & & & - & $0.23 \quad(-0.40,0.86)$ & 5.57 \\
\hline Vukomanovic 2016 & 50 & $55.0 \pm 7.0$ & $52.00 \%$ & $7.3 \pm 1.5$ & $29.1 \pm 3.3$ & 40 & $50.9 \pm 9.0$ & $55.00 \%$ & $5.2 \pm 0.4$ & $24.1 \pm 2.8$ & & $\leftarrow$ & $0.19 \quad(-0.22,0.61)$ & 6.02 \\
\hline Wang 2006, T2DM with hypertension / non T2DM with hypertension & 33 & 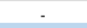 & $100 \%$ & . & - & 36 & 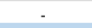 & $100 \%$ & & & & & $3.84 \quad(3.04,4.65)$ & 5.13 \\
\hline Overall (I-squared $=90.1 \%, p=0.914$ ) & & & & & & & & & & & & $>$ & $0.02(-0.38,0.43)$ & 100 \\
\hline
\end{tabular}

Fig 9. Meta-analysis of LF/HF of type 2 diabetes mellitus patients compared with controls. -: non reported data (missing SD were also non reported). 95\% CI: 95\% confident intervals; BMI: Body Mass Index; LF/HF: Low Frequency/High Frequency ratio; T2DM: Type 2 Diabetes Mellitus; SD: Standard Deviation. 


\begin{tabular}{|c|c|c|c|c|c|c|c|}
\hline \multirow{2}{*}{$\begin{array}{ll} & \text { Variables } \\
\text { RR }\end{array}$} & \multirow[t]{2}{*}{$\begin{array}{c}\text { Number of } \\
\text { studies included }\end{array}$} & \multirow[t]{2}{*}{$\begin{array}{c}\text { I- } \\
\text { squared }\end{array}$} & & & \multicolumn{2}{|c|}{ Coefficient (95\% Cl) } & p-value \\
\hline & & & & & & & \\
\hline Age, years & 12 & $98.2 \%$ & 0 & . & 9 & $(-24,42)$ & 0.550 \\
\hline Sex, male as reference & 12 & $99.5 \%$ & d & 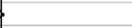 & -17 & $(-40,5.4)$ & 0.121 \\
\hline $\mathrm{HbA} 1 \mathrm{c}, \%$ & 7 & $94.0 \%$ & $\infty$ & & -383 & $(-756,-8.8)$ & 0.046 \\
\hline Body mass index, kg.m-2 & 6 & $98.3 \%$ & $\longrightarrow$ & - & -157 & $(-430,116)$ & 0.186 \\
\hline Systolic blood pressure, $\mathrm{cmHg}$ & 6 & $98.0 \%$ & $-\infty$ & & -380 & $(-703,-53)$ & 0.032 \\
\hline Diastolic blood pressure, $\mathrm{cmHg}$ & 6 & $98.2 \%$ & & & -1534 & $(-3881,813)$ & 0.144 \\
\hline SDNN & & & & & & & \\
\hline Age, years & 25 & $89.5 \%$ & i & 3 & 1.7 & $(-1.5,4.9)$ & 0.279 \\
\hline Sex, male as reference & 25 & $88.5 \%$ & 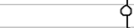 & 3 & 0.1 & $(-1.2,1.5)$ & 0.836 \\
\hline Time from diagnosis, years & 15 & $82.6 \%$ & & 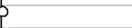 & 10 & $(2.1,18)$ & 0.018 \\
\hline Blood glucose levels, $10 \mathrm{mg} / \mathrm{dl}$ & 18 & $61.8 \%$ & & $p$ & 24 & $(17,30)$ & $<0.001$ \\
\hline Systolic blood pressure, $\mathrm{cmHg}$ & 16 & $86.9 \%$ & & . & 6.4 & $(-20,32)$ & 0.603 \\
\hline Diastolic blood pressure, $\mathrm{cmHg}$ & 16 & $89.1 \%$ & -0 & 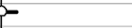 & 5.5 & $(-52,63)$ & 0.840 \\
\hline $\mathrm{HbA} 1 \mathrm{c}, \%$ & 19 & $89.3 \%$ & & . & 10 & $(-18,39)$ & 0.453 \\
\hline Body mass index, kg.m-2 & 15 & $90.2 \%$ & 0 & 3 & 2.6 & $(-8.8,14)$ & 0.634 \\
\hline Total cholesterol, g/l & 12 & $90.3 \%$ & $\rightarrow$ & - & 25 & $(-150,201)$ & 0.759 \\
\hline LDL cholesterol, g/l & 10 & $90.2 \%$ & & - & -11 & $(-465,443)$ & 0.101 \\
\hline HDL cholesterol, g/l & 10 & $83.7 \%$ & & - & 1251 & $(292,2210)$ & 0.017 \\
\hline Triglycerides, $\mathrm{g} / \mathrm{l}$ & 17 & $91.4 \%$ & - & - & 14 & $(-59,88)$ & 0.688 \\
\hline pNN50 & & & & & & & \\
\hline Age, years & 14 & $0.0 \%$ & 0 & 3 & 0.2 & $(-0.2,0.5)$ & 0.316 \\
\hline Sex, Male as reference & 14 & $0.0 \%$ & 0 & 3 & 0.0 & $(-0.1,0.1)$ & 0.462 \\
\hline Time from diagnosis, years & 9 & $0.0 \%$ & 0 & 3 & 0.3 & $(-0.9,1.4)$ & 0.587 \\
\hline Blood glucose levels, $10 \mathrm{mg} / \mathrm{dl}$ & 11 & $0.0 \%$ & 0 & 3 & -1.0 & $(-2.9,1)$ & 0.313 \\
\hline Systolic blood pressure, $\mathrm{cmHg}$ & 9 & $0.0 \%$ & 0 & 3 & -1.0 & $(-3,1)$ & 0.263 \\
\hline Diastolic blood pressure, $\mathrm{cmHg}$ & 9 & $0.0 \%$ & o & 3 & -2.3 & $(-6.2,1.6)$ & 0.203 \\
\hline Total cholesterol, g/l & 6 & $0.0 \%$ & & . & 15 & $(-13,43)$ & 0.201 \\
\hline Triglycerides, $\mathrm{g} / \mathrm{L}$ & 11 & $0.0 \%$ & t & T & -2.4 & $(-8.4,3.7)$ & 0.401 \\
\hline RMSSD & & & & & & & \\
\hline Age, years & 21 & $95.2 \%$ & o & 3 & -0.1 & $(-1.3,1.1)$ & 0.820 \\
\hline Sex, Male as reference & 21 & $93.9 \%$ & 0 & 3 & 0.1 & $(-0.3,0.4)$ & 0.787 \\
\hline Time from diagnosis, years & 12 & $87.3 \%$ & \$ & 3 & 3.0 & $(-1,7)$ & 0.121 \\
\hline Blood glucose levels, $10 \mathrm{mg} / \mathrm{dl}$ & 13 & $60.0 \%$ & 0 & 3 & 4.2 & $(1.7,6.6)$ & 0.003 \\
\hline $\mathrm{HbA} 1 \mathrm{c}, \%$ & 15 & $63.9 \%$ & t & S & 2.8 & $(-5.1,11)$ & 0.451 \\
\hline Body mass index, kg.m-2 & 16 & $93.2 \%$ & I & 3 & 0.9 & $(-1.5,3.2)$ & 0.428 \\
\hline Systolic blood pressure, $\mathrm{cmHg}$ & 13 & $96.4 \%$ & o & S & 0.3 & $(-6.7,7.3)$ & 0.934 \\
\hline Diastolic blood pressure, $\mathrm{cmHg}$ & 13 & $94.1 \%$ & S & 3 & -2.1 & $(-19,15)$ & 0.783 \\
\hline LDL cholesterol, g/L & 8 & $79.0 \%$ & J & & -6.9 & $(-172,159)$ & 0.922 \\
\hline HDL cholesterol, g/L & 8 & $24.2 \%$ & & $\infty$ & 246 & $(186,307)$ & $<0.001$ \\
\hline Triglycerides, $g / L$ & 13 & $95.5 \%$ & d & 3 & 2.7 & $(-16,21)$ & 0.751 \\
\hline Total Power & & & & & & & \\
\hline Age, years & 15 & $78.6 \%$ & d & 3 & 0.1 & $(-0.6,0.8)$ & 0.762 \\
\hline Sex, Male as reference & 15 & $78.5 \%$ & T & 3 & 0.0 & $(-0.1,0.1)$ & 0.843 \\
\hline Time from diagnosis, years & 8 & $66.0 \%$ & 7 & & -1214 & $(-2129,-299)$ & 0.021 \\
\hline $\mathrm{HbA} 1 \mathrm{c}, \%$ & 10 & $69.1 \%$ & d & 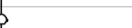 & 4.5 & $(-13,22)$ & 0.563 \\
\hline Body mass index, kg.m-2 & 10 & $79.3 \%$ & T & 5 & 1.1 & $(-1.6,3.9)$ & 0.364 \\
\hline Systolic blood pressure, $\mathrm{cmHg}$ & 9 & $84.4 \%$ & & . & 8.3 & $(-17,33)$ & 0.456 \\
\hline Diastolic blood pressure, $\mathrm{cmHg}$ & 9 & $84.3 \%$ & t & 5 & 5.2 & $(-10,20)$ & 0.431 \\
\hline Triglycerides, g/L & 6 & $64.2 \%$ & T & T & 2.1 & $(-15,19)$ & 0.748 \\
\hline LF & & & & & & & \\
\hline Age, years & 22 & $80.5 \%$ & d & 3 & -0.7 & $(-1.0,-0.4)$ & $<0.001$ \\
\hline Sex, Male as reference & 22 & $67.8 \%$ & S & 3 & -0.3 & $(-0.4,-0.1)$ & 0.006 \\
\hline Time from diagnosis, years & 17 & $86.7 \%$ & o & 3 & -0.6 & $(-1.5,0.3)$ & 0.173 \\
\hline Blood glucose levels, $10 \mathrm{mg} / \mathrm{dl}$ & 8 & $0.0 \%$ & & $\infty$ & 112 & $(31,193)$ & 0.015 \\
\hline $\mathrm{HbA} 1 \mathrm{c}, \%$ & 19 & $85.6 \%$ & o & 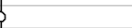 & -2.1 & $(-11,6.5)$ & 0.613 \\
\hline Body mass index, kg.m-2 & 15 & $86.8 \%$ & T & & 0.8 & $(0.3,1.3)$ & 0.003 \\
\hline Systolic blood pressure, $\mathrm{cmHg}$ & 16 & $91.4 \%$ & I & & -3.4 & $(-6.9,0.2)$ & 0.062 \\
\hline Diastolic blood pressure, $\mathrm{cmHg}$ & 16 & $92.4 \%$ & a & 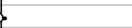 & -11 & $(-35,14)$ & 0.362 \\
\hline Total cholesterol, g/L & 7 & $21.8 \%$ & & - & 1133 & $(408,1858)$ & 0.010 \\
\hline $\mathrm{HF}$ & & & & & & & \\
\hline Age, years & 23 & $70.8 \%$ & d & & -0.6 & $(-0.9,-0.3)$ & $<0.001$ \\
\hline Sex, Male as reference & 23 & $67.8 \%$ & s & & -0.3 & $(-0.4,-0.1)$ & 0.006 \\
\hline Time from diagnosis, years & 18 & $79.8 \%$ & 0 & & -0.5 & $(-1.2,0.2)$ & 0.150 \\
\hline Blood glucose levels, $10 \mathrm{mg} / \mathrm{dl}$ & 9 & $0.0 \%$ & & a. & 34 & $(6,63)$ & 0.024 \\
\hline $\mathrm{HbA} 1 \mathrm{c}, \%$ & 20 & $78.1 \%$ & 8 & & -0.8 & $(-7.5,5.8)$ & 0.792 \\
\hline Body mass index, kg.m-2 & 16 & $81.8 \%$ & 8 & & 0.7 & $(0.3,1.1)$ & 0.005 \\
\hline Systolic blood pressure, $\mathrm{cmHg}$ & 17 & $87.0 \%$ & o & & -3.1 & $(-6.2,-0.02)$ & 0.049 \\
\hline Diastolic blood pressure, $\mathrm{cmHg}$ & 17 & $89.0 \%$ & d & & -1.0 & $(-3.1,1.2)$ & 0.347 \\
\hline Total cholesterol, g/L & 7 & $20.1 \%$ & & & 490 & $(100,879)$ & 0.023 \\
\hline Triglycerides, $\mathrm{g} / \mathrm{L}$ & 9 & $19.4 \%$ & 1 & ? & 1.3 & $(-7.4,10.1)$ & 0.730 \\
\hline LF / HF & & & & & & & \\
\hline Age, years & 16 & $0.0 \%$ & S & & -0.03 & $(-0.1,0.1)$ & 0.965 \\
\hline Sex, Male as reference & 16 & $0.0 \%$ & 9 & & 0.01 & $(-0.02,0.03)$ & 0.367 \\
\hline Time from diagnosis, years & 11 & $0.0 \%$ & 8 & & -0.05 & $(-0.2,0.1)$ & 0.395 \\
\hline Blood glucose levels, $10 \mathrm{mg} / \mathrm{dl}$ & 12 & $0.0 \%$ & O & & 0.10 & $(-0.2,0.4)$ & 0.472 \\
\hline $\mathrm{HbA} 1 \mathrm{c}, \%$ & 13 & $0.0 \%$ & 0 & & 0.60 & $(-0.1,1.3)$ & 0.095 \\
\hline Body mass index, kg.m-2 & 7 & $0.0 \%$ & o & & 0.09 & $(-0.3,0.4)$ & 0.562 \\
\hline Systolic blood pressure, $\mathrm{cmHg}$ & 10 & $0.0 \%$ & o & & 0.10 & $(-0.7,0.9)$ & 0.707 \\
\hline Diastolic blood pressure, $\mathrm{cmHg}$ & 10 & $0.0 \%$ & 8 & & 0.20 & $(-0.2,0.6)$ & 0.324 \\
\hline Total cholesterol, g/L & 9 & $0.0 \%$ & 0 & & 0.70 & $(-2.1,3.6)$ & 0.561 \\
\hline Triglycerides, $\mathrm{g} / \mathrm{l}$ & 7 & $0.0 \%$ & d & & 0.10 & $(-3.2,3.5)$ & 0.923 \\
\hline
\end{tabular}

Fig 10. Meta-regression of factors influencing heart rate variability in type 2 diabetes mellitus. $95 \%$ CI: $95 \%$ confident intervals; RR: RR intervals; SDNN: Standard Deviation of RR intervals; pNN50: percentage of RR intervals with more than 50 ms variation; RMSSD: square root of mean squared differences of successive RR intervals; TP: Total Power; LF: Low Frequency; HF: High Frequency; LF/HF: Low Frequency/High Frequency ratio.

https://doi.org/10.1371/journal.pone.0195166.g010 
Dyslipdaemia and high blood pressure are linked with a decreased HRV in T2DM. Finally, age and the male gender were associated with both a decrease in sympathetic and parasympathetic activity.

\section{An alteration of both sympathetic and parasympathetic activity}

Adaptation to stress is characterized by an increase in sympathetic activity and a decrease in parasympathetic activity, inducing a state of alertness [17]. Interestingly, common diseases such as rheumatoid arthritis [67], depression [68], schizophrenia [69], multiple sclerosis [70], active ulcerative colitis [71], obesity and metabolic syndrome [72,73], myocardial infarctions [74], high blood pressure [75], smoking [76], and cancer [77], are associated with a decrease in parasympathetic activity and an activation of sympathetic activity. However, we demonstrated that T2DM patients had a decrease in both parasympathetic and sympathetic activity. An explanation could be that T2DM is a metabolic disease responsible for a cardiac autonomic neuropathy that affects both sympathetic and parasympathetic fibers. With the exception of the LF/HF ratio, which did not differ in our meta-analysis due to comparable changes in the LF and HF components, T2DM has a negative influence on almost all HRV parameters, reflecting the fact that diabetes leads to a cardiac autonomic dysfunction.

\section{Deleterious effects of altered glucose metabolism}

The significant relationship between altered glucose metabolism and HRV may explain the deleterious general metabolic effects on both parasympathetic and sympathetic activity. Interestingly, blood glucose levels were associated with both an increase in LF (sympathetic) and HF (parasympathetic), as well as SDNN (sympathetic) and RMSSD (parasympathetic), which may appear contradictory. In healthy individuals, parasympathetic activity is triggered by an increase in blood glucose levels through insulin responses [78]. Unfortunately, insulin levels were not reported in most studies included in our meta-analysis, which precluded further analysis. Another explanation of this relation that could appear contradictory is that blood glucose levels are not a good marker to evaluate diabetes control compared with $\mathrm{HbA1c}$ : blood glucose levels reflect an instantaneous level whereas $\mathrm{HbA1c}$ reflect diabetes control over the previous months. In almost all included studies, patients had blood sampling during hospitalisation, after a steady state and in a controlled environment for diet with a close monitoring of capillary blood glucose, which may explain our contradictory results. Another convincing point is that the relationships between HbAlc and HRV is logic. We demonstrated that higher levels of HbAlc were associated with shorter RR intervals, which were associated with an increased risk of ventricular arrhythmias [79]. There was also a tendency for higher LF/HF ratio (i.e. decreased HRV) with higher levels of HbA1c. Furthermore, time from diagnosis of T2DM was linked with a higher level of SDNN. Despite we demonstrated a decreased SDNN in T2DM patients, the metaregression is not contradictory but may simply highlights the fact that cardiac parasympathetic activity in T2DM is affected before sympathetic activity [80]. Time from diagnosis of T2DM was also linked with a lower level of total power, but not with LF and HF. Thus we can hypothesize that very low frequencies (VLF) could be decreased in T2DM. No studies included in our meta-analysis reported VLF. Although less commonly used, VLF are recognised as the most powerful independent predictors of mortality in patients with heart failure or in patients with chronic haemodialysis [81]. Despite few studies assessing VLF in diabetes, interesting relationships were reported between VLF and sleep apnea in diabetics [82]. The potential significance of VLF in diabetes should be studied further. 


\section{Other variables linked with HRV in T2DM}

Similarly, total cholesterol was associated with both an increase in LF and HF, and HDL was associated with an increase in SDNN and RMSSD. To our knowledge, there is no data on hypercholesterolemia and HRV in the literature. Interestingly, some studies showed that a decrease in LDL by statin therapy could improve HRV parameters $[83,84]$. We demonstrated that an increase in systolic blood pressure was linked with shorter RR intervals and a decrease in HF. Despite no study previously assessing this relationship in diabetes, conflicting results were reported in the general population, with either high blood pressure associated with an increase in all spectral parameters [85], or a decrease in HRV [86]. It has also been suggested that the decrease in autonomic nervous function precedes the development of clinical hypertension [87]. Moreover, we found a significant relationship between BMI and HRV. Such relationships have been either found $[88,89]$ or not $[90,91]$ in the literature. However, the severity of obesity-related diseases is not directly linked to the accumulation of total body fat but rather to its distribution, and particularly to visceral localization [92]. HRV parameters have been previously correlated with sagittal abdominal diameter, anterior forearm skinfold thickness [93] and waist-hip ratio [90]. HRV parameters can also be improved after weight loss [94]. Finally, in line with the literature, we demonstrated a decrease in both LF and HF with age [95] and the male gender [96]. However, age and gender have a minor role on HRV parameters compared with the variables linked to T2DM.

\section{Limitations}

All meta-analyses have limitations [97]. Meta-analyses inherit the limitations of the individual studies of which they are composed and are subjected to a bias of selection of included studies. However, the use of broader keywords in the search strategy limits the number of missing studies. Despite our rigorous criteria for including studies in our meta-analysis, their quality varied. Indeed, most of the studies included were cross-sectional, with different measurement conditions for HRV parameters associated with a high inter and intra-individual variability. We demonstrated that all parameters measuring HRV were significantly decreased in T2DM compared with controls. Though there were similarities between the participants' inclusion criteria, they were not identical. Moreover, the health status of controls was not detailed in all studies, which could have influenced HRV parameters. This may have also minimized the differences in HRV between T2DM patients and controls. In addition, some studies were monocentric, limiting the generalizability of our results. However, included studies were homogeneous according to funnel plots, and the populations investigated in the meta-analysis appeared to be equally distributed around the world. Similarly, the final number of patients included in the metaanalysis was not very high and may precluded generalizability, however the mean age could be considered as quite representative. Despite missing data, our metaregressions demonstrated significant and interesting relationships, particularly between HRV parameters and variables linked with T2DM. Variables retrieved from declarative data in each study included are also a putative bias.

\section{Conclusion}

We reported strong evidence for an overall decrease in HRV in T2DM patients. Both sympathetic and parasympathetic activity were decreased, which can be explained by the deleterious effects of altered glucose metabolism on HRV. The benefits of an HRV evaluation in assessing and monitoring the severity of T2DM should be further studied, given its potential as a noninvasive, reliable and pain-free measurement. 


\section{Supporting information}

S1 Fig. Quality score of included articles.

(TIF)

S1 Appendix. Example of search strategy on PubMed database.

(PDF)

S2 Appendix. PRISMA checklist.

(DOCX)

\section{Author Contributions}

Conceptualization: Daniela Pfabigan, Salwan Maqdasy, Frédéric Dutheil.

Data curation: Frédéric Dutheil.

Formal analysis: Bruno Pereira, Frédéric Dutheil.

Investigation: Thomas Benichou, Frédéric Dutheil.

Methodology: Frédéric Dutheil.

Project administration: Frédéric Dutheil.

Resources: Thomas Benichou, Frédéric Dutheil.

Software: Thomas Benichou, Bruno Pereira, Frédéric Dutheil.

Supervision: Frédéric Dutheil.

Validation: Martial Mermillod, Igor Tauveron, Daniela Pfabigan, Salwan Maqdasy, Frédéric Dutheil.

Visualization: Martial Mermillod, Igor Tauveron, Daniela Pfabigan, Salwan Maqdasy, Frédéric Dutheil.

Writing - original draft: Thomas Benichou, Frédéric Dutheil.

Writing - review \& editing: Martial Mermillod, Igor Tauveron, Daniela Pfabigan, Salwan Maqdasy.

\section{References}

1. da Rocha Fernandes J, Ogurtsova K, Linnenkamp U, Guariguata L, Seuring T, Zhang P, et al. IDF Diabetes Atlas estimates of 2014 global health expenditures on diabetes. Diabetes Res Clin Pract. 2016; 117: 48-54. https://doi.org/10.1016/j.diabres.2016.04.016 PMID: 27329022

2. Meigs JB. Epidemiology of type 2 diabetes and cardiovascular disease: translation from population to prevention: the Kelly West award lecture 2009. Diabetes Care. 2010; 33: 1865-1871. https://doi.org/10. 2337/dc10-0641 PMID: 20668155

3. Jaacks LM, Siegel KR, Gujral UP, Narayan KMV. Type 2 diabetes: A 21st century epidemic. Best Pract Res Clin Endocrinol Metab. 2016; 30: 331-343. https://doi.org/10.1016/j.beem.2016.05.003 PMID: 27432069

4. Ginter E, Simko V. Global prevalence and future of diabetes mellitus. Adv Exp Med Biol. 2012; 771: 3541. PMID: 23393669

5. Ginter E, Simko V. Type 2 diabetes mellitus, pandemic in 21st century. Adv Exp Med Biol. 2012; 771 : 42-50. PMID: 23393670

6. Colberg SR, Sigal RJ, Fernhall B, Regensteiner JG, Blissmer BJ, Rubin RR, et al. Exercise and Type 2 Diabetes: The American College of Sports Medicine and the American Diabetes Association: joint position statement. Diabetes Care. 2010; 33: e147-e167. https://doi.org/10.2337/dc10-9990 PMID: 21115758 
7. Fox CS. Cardiovascular Disease Risk Factors, Type 2 Diabetes Mellitus, and the Framingham Heart Study. Trends Cardiovasc Med. 2010; 20: 90-95. https://doi.org/10.1016/j.tcm.2010.08.001 PMID: 21130952

8. Laakso M. Cardiovascular Disease in Type 2 Diabetes From Population to Man to Mechanisms: The Kelly West Award Lecture 2008. Diabetes Care. 2010; 33: 442-449. https://doi.org/10.2337/dc09-0749 PMID: 20103560

9. Tancredi M, Rosengren A, Svensson A-M, Kosiborod M, Pivodic A, Gudbjörnsdottir S, et al. Excess Mortality among Persons with Type 2 Diabetes. N Engl J Med. 2015; 373: 1720-1732. https://doi.org/ 10.1056/NEJMoa1504347 PMID: 26510021

10. Shah AD, Langenberg C, Rapsomaniki E, Denaxas S, Pujades-Rodriguez M, Gale CP, et al. Type 2 diabetes and incidence of cardiovascular diseases: a cohort study in 1.9 million people. Lancet Diabetes Endocrinol. 2015; 3: 105-113. https://doi.org/10.1016/S2213-8587(14)70219-0 PMID: 25466521

11. Ponto KA, Koenig J, Peto T, Lamparter J, Raum P, Wild PS, et al. Prevalence of diabetic retinopathy in screening-detected diabetes mellitus: results from the Gutenberg Health Study (GHS). Diabetologia. 2016; 59: 1913-1919. https://doi.org/10.1007/s00125-016-4013-5 PMID: 27314413

12. Ksvh Kumar, Modi K, Basile A, Kota S. Profile of microvascular disease in type 2 diabetes in a tertiary health care hospital in India. Ann Med Health Sci Res. 2012; 2: 103. https://doi.org/10.4103/2141-9248. 105654 PMID: 23439986

13. Hazari A, Maiya AG, Shivashankara KN, Agouris I, Monteiro A, Jadhav R, et al. Kinetics and kinematics of diabetic foot in type 2 diabetes mellitus with and without peripheral neuropathy: a systematic review and meta-analysis. SpringerPlus. 2016; 5: 1819. https://doi.org/10.1186/s40064-016-3405-9 PMID: 27812455

14. Singh JP, Larson MG, O'Donnell CJ, Wilson PF, Tsuji H, Lloyd-Jones DM, et al. Association of hyperglycemia with reduced heart rate variability (The Framingham Heart Study). Am J Cardiol. 2000; 86: 309-312. PMID: 10922439

15. Ziegler D, Zentai CP, Perz S, Rathmann W, Haastert B, Döring A, et al. Prediction of mortality using measures of cardiac autonomic dysfunction in the diabetic and nondiabetic population: the MONICA/ KORA Augsburg Cohort Study. Diabetes Care. 2008; 31: 556-561. https://doi.org/10.2337/dc07-1615 PMID: 18086873

16. Ewing DJ, Martyn CN, Young RJ, Clarke BF. The value of cardiovascular autonomic function tests: 10 years experience in diabetes. Diabetes Care. 1985; 8: 491-498. PMID: 4053936

17. Hufnagel $C$, Chambres $P$, Bertrand PR, Dutheil F. The Need for Objective Measures of Stress in Autism. Front Psychol. 2017; 8. https://doi.org/10.3389/fpsyg.2017.00064 PMID: 28191002

18. Boudet G, Walther G, Courteix D, Obert P, Lesourd B, Pereira B, et al. Paradoxical dissociation between heart rate and heart rate variability following different modalities of exercise in individuals with metabolic syndrome: The RESOLVE study. Eur J Prev Cardiol. 2017; 24: 281-296. https://doi.org/10. 1177/2047487316679523 PMID: 27856807

19. Dutheil F, Chambres P, Hufnagel C, Auxiette C, Chausse P, Ghozi R, et al. "Do Well B.": Design Of WELL Being monitoring systems. A study protocol for the application in autism. BMJ Open. 2015; 5: e007716-e007716. https://doi.org/10.1136/bmjopen-2015-007716 PMID: 25710916

20. Solanki JD, Basida SD, Mehta HB, Panjwani SJ, Gadhavi BP. Comparative study of cardiac autonomic status by heart rate variability between under-treatment normotensive and hypertensive known type 2 diabetics. Indian Heart J. https://doi.org/10.1016/j.ihj.2016.07.013 PMID: 28228307

21. Lotric MB, Stefanovska A, Stajer D, Urbancic-Rovan V. Spectral components of heart rate variability determined by wavelet analysis. Physiol Meas. 2000; 21: 441-457. PMID: 11110243

22. Roy $B$, Ghatak $S$. Nonlinear methods to assess changes in heart rate variability in type 2 diabetic patients. Arq Bras Cardiol. 2013; 101: 317-327. https://doi.org/10.5935/abc.20130181 PMID: 24008652

23. Gerritsen J, Dekker JM, TenVoorde BJ, Kostense PJ, Heine RJ, Bouter LM, et al. Impaired autonomic function is associated with increased mortality, especially in subjects with diabetes, hypertension, or a history of cardiovascular disease the Hoorn study. Diabetes Care. 2001; 24: 1793-1798. PMID: 11574444

24. Zhang J. Effect of Age and Sex on Heart Rate Variability in Healthy Subjects. J Manipulative Physio Ther. 2007; 30: 374-379. https://doi.org/10.1016/j.jmpt.2007.04.001 PMID: 17574955

25. Saleem S, Hussain MM, Majeed SMI, Khan MA. Gender differences of heart rate variability in healthy volunteers. JPMA J Pak Med Assoc. 2012; 62: 422-425. PMID: 22755301

26. Rothberg LJ, Lees T, Clifton-Bligh R, Lal S. Association Between Heart Rate Variability Measures and Blood Glucose Levels: Implications for Noninvasive Glucose Monitoring for Diabetes. Diabetes Technol Ther. 2016; 18: 366-376. https://doi.org/10.1089/dia.2016.0010 PMID: 27258123 
27. Kilit C, Demir S, Pasali Kilit T, Melek M. The effects of metformin and rosiglitazone on heart rate variability in type 2 diabetes mellitus. Int Soc Electrocardiol Annu Meet-Istanb 200734th Int Congr Electrocardiol. 2007;40: S40-S41.

28. Kumarathurai $\mathrm{P}$, Anholm C, Larsen BS, Olsen RH, Madsbad S, Kristiansen O, et al. Effects of Liraglutide on Heart Rate and Heart Rate Variability: A Randomized, Double-Blind, Placebo-Controlled Crossover Study. Diabetes Care. 2016; dc161580. https://doi.org/10.2337/dc16-1580 PMID: 27797930

29. Heart rate variability: standards of measurement, physiological interpretation and clinical use. Task Force of the European Society of Cardiology and the North American Society of Pacing and Electrophysiology. Circulation. 1996; 93: 1043-1065. PMID: 8598068

30. da Costa BR, Cevallos M, Altman DG, Rutjes AWS, Egger M. Uses and misuses of the STROBE statement: bibliographic study. BMJ Open. 2011; 1: e000048. https://doi.org/10.1136/bmjopen-2010000048 PMID: 22021739

31. Elm E v., Altman DG, Egger M, Pocock SJ, Gotzsche PC, Vandenbroucke JP, et al. Strengthening the reporting of observational studies in epidemiology (STROBE) statement: guidelines for reporting observational studies. BMJ. 2007; 335: 806-808. https://doi.org/10.1136/bmj.39335.541782.AD PMID: 17947786

32. Moher D, Hopewell S, Schulz KF, Montori V, Gotzsche PC, Devereaux PJ, et al. CONSORT 2010 Explanation and Elaboration: updated guidelines for reporting parallel group randomised trials. BMJ. 2010; 340: c869-c869. https://doi.org/10.1136/bmj.c869 PMID: 20332511

33. Otzenberger H, Gronfier C, Simon C, Charloux A, Ehrhart J, Piquard F, et al. Dynamic heart rate variability: a tool for exploring sympathovagal balance continuously during sleep in men. Am J Physiol. 1998; 275: H946-950. PMID: 9724299

34. Benoist d'Azy C, Pereira B, Naughton G, Chiambaretta F, Dutheil F. Antibioprophylaxis in Prevention of Endophthalmitis in Intravitreal Injection: A Systematic Review and Meta-Analysis. PloS One. 2016; 11: e0156431. https://doi.org/10.1371/journal.pone.0156431 PMID: 27257676

35. Benoist d'Azy C, Pereira B, Chiambaretta F, Dutheil F. Oxidative and Anti-Oxidative Stress Markers in Chronic Glaucoma: A Systematic Review and Meta-Analysis. PloS One. 2016; 11: e0166915. https:// doi.org/10.1371/journal.pone.0166915 PMID: 27907028

36. Courtin R, Pereira B, Naughton G, Chamoux A, Chiambaretta F, Lanhers C, et al. Prevalence of dry eye disease in visual display terminal workers: a systematic review and meta-analysis. BMJ Open. 2016; 6: e009675. https://doi.org/10.1136/bmjopen-2015-009675 PMID: 26769784

37. Ollier M, Chamoux A, Naughton G, Pereira B, Dutheil F. Chest CT scan screening for lung cancer in asbestos occupational exposure: a systematic review and meta-analysis. Chest. 2014; 145: 13391346. https://doi.org/10.1378/chest.13-2181 PMID: 24480869

38. Lanhers C, Pereira B, Naughton G, Trousselard M, Lesage F-X, Dutheil F. Creatine Supplementation and Upper Limb Strength Performance: A Systematic Review and Meta-Analysis. Sports Med. 2017; 47: 163-173. https://doi.org/10.1007/s40279-016-0571-4 PMID: 27328852

39. Lanhers C, Pereira B, Naughton G, Trousselard M, Lesage F-X, Dutheil F. Creatine Supplementation and Lower Limb Strength Performance: A Systematic Review and Meta-Analyses. Sports Med. 2015; 45: 1285-1294. https://doi.org/10.1007/s40279-015-0337-4 PMID: 25946994

40. DerSimonian R, Laird N. Meta-analysis in clinical trials. Control Clin Trials. 1986; 7: 177-188. PMID: 3802833

41. Citrome L, Magnusson K. Paging Dr Cohen, Paging Dr Cohen... An effect size interpretation is required STAT!: visualising effect size and an interview with Kristoffer Magnusson. Int J Clin Pract. 2014; 68: 533-534. https://doi.org/10.1111/ijcp.12435 PMID: 24750523

42. Porojan M, Costin S, Poantă L, Cerghizan A, Pop D, Dumitraşcu DL. Autonomic neuropathy and plasma catecholamine in patients with diabetes mellitus. Romanian J Intern Med Rev Roum Médecine Interne. 2010; 48: 341-345.

43. Hathaway DK, Cashion AK, Wicks MN, Milstead EJ, Gaber AO. Cardiovascular dysautonomia of patients with end-stage renal disease and type I or type II diabetes. Nurs Res. 1998; 47: 171-179. PMID: 9610651

44. Kondo K, Matsubara T, Nakamura J, Hotta N. Characteristic patterns of circadian variation in plasma catecholamine levels, blood pressure and heart rate variability in Type 2 diabetic patients. Diabet Med J Br Diabet Assoc. 2002; 19: 359-365.

45. Perciaccante A, Fiorentini A, Paris A, Serra P, Tubani L. Circadian rhythm of the autonomic nervous system in insulin resistant subjects with normoglycemia, impaired fasting glycemia, impaired glucose tolerance, type 2 diabetes mellitus. BMC Cardiovasc Disord. 2006; 6: 19. https://doi.org/10.1186/14712261-6-19 PMID: 16670002 
46. Giordano M, Manzella D, Paolisso G, Caliendo A, Varricchio M, Giordano C. Differences in heart rate variability parameters during the post-dialytic period in type II diabetic and non-diabetic ESRD patients. Nephrol Dial Transplant Off Publ Eur Dial Transpl Assoc-Eur Ren Assoc. 2001; 16: 566-573.

47. Rasic-Milutinovic ZR, Milicevic DR, Milovanovic BD, Perunicic-Pekovic GB, Pencic BD. Do components of metabolic syndrome contribute to cardiac autonomic neuropathy in non-diabetic patients? Saudi Med J. 2010; 31: 650-657. PMID: 20563363

48. Burger AJ, Aronson D. Effect of diabetes mellitus on heart rate variability in patients with congestive heart failure. Pacing Clin Electrophysiol PACE. 2001; 24: 53-59. PMID: 11227970

49. Yasuda G, Hasegawa K, Kuji T, Ogawa N, Shimura G, Umemura S, et al. Effects of doxazosin on ambulatory blood pressure and sympathetic nervous activity in hypertensive Type 2 diabetic patients with overt nephropathy. Diabet Med J Br Diabet Assoc. 2005; 22: 1394-1400. https://doi.org/10.1111/j. 1464-5491.2005.01636.x PMID: 16176202

50. Wang G. Effects of impaired glucose metabolism on heart rate variability and blood pessure variability in essential hpertensive patients. J Huazhong Univ Sci Technol. 2006; 26: 654-656.

51. Poanta L, Porojan M, Dumitrascu DL. Heart rate variability and diastolic dysfunction in patients with type 2 diabetes mellitus. Acta Diabetol. 2011; 48: 191-196. https://doi.org/10.1007/s00592-011-0256-2 PMID: 21298295

52. Mylonopoulou M, Tentolouris N, Antonopoulos S, Mikros S, Katsaros K, Melidonis A, et al. Heart rate variability in advanced chronic kidney disease with or without diabetes: midterm effects of the initiation of chronic haemodialysis therapy. Nephrol Dial Transplant Off Publ Eur Dial Transpl Assoc-Eur Ren Assoc. 2010; 25: 3749-3754. https://doi.org/10.1093/ndt/gfq226 PMID: 20466659

53. Comunello A, Dassie F, Martini C, De Carlo E, Mioni R, Battocchio M, et al. Heart rate variability is reduced in acromegaly patients and improved by treatment with somatostatin analogues. Pituitary. 2015; 18: 525-534. https://doi.org/10.1007/s11102-014-0605-6 PMID: 25261332

54. Istenes I, Körei AE, Putz Z, Németh N, Martos T, Keresztes K, et al. Heart rate variability is severely impaired among type 2 diabetic patients with hypertension. Diabetes Metab Res Rev. 2014; 30: 305312. PMID: 24829967

55. Nagy K, Sipos E, El Hadj Othmane T. [Heart rate variability is significantly reduced in non-diabetic patients with hypertension]. Orv Hetil. 2014; 155: 865-870. https://doi.org/10.1556/OH.2014.29886 PMID: 24860051

56. Eguchi K, Schwartz JE, Pickering TG, Hoshide S, Ishikawa J, Shimada K, et al. Increased heart rate variability during sleep is a predictor for future cardiovascular events in patients with type 2 diabetes. Hypertens Res Off J Jpn Soc Hypertens. 2010; 33: 737-742. https://doi.org/10.1038/hr.2010.61 PMID: 20431592

57. Vora R, Zareba W, Utell MJ, Pietropaoli AP, Chalupa D, Little EL, et al. Inhalation of ultrafine carbon particles alters heart rate and heart rate variability in people with type 2 diabetes. Part Fibre Toxicol. 2014; 11: 31. https://doi.org/10.1186/s12989-014-0031-y PMID: 25028096

58. Boer-Martins L, Figueiredo VN, Demacq C, Martins LC, de Faria APC, Moraes Cde H, et al. Leptin and aldosterone in sympathetic activity in resistant hypertension with or without type 2 diabetes. Arq Bras Cardiol. 2012; 99: 642-648. PMID: 22641394

59. Soydan N, Bretzel RG, Fischer B, Wagenlehner F, Pilatz A, Linn T. Reduced capacity of heart rate regulation in response to mild hypoglycemia induced by glibenclamide and physical exercise in type 2 diabetes. Metabolism. 2013; 62: 717-724. https://doi.org/10.1016/j.metabol.2012.12.003 PMID: 23318051

60. Molon G, Costa A, Bertolini L, Zenari L, Arcaro G, Barbieri E, et al. Relationship between abnorma microvolt T-wave alternans and poor glycemic control in type 2 diabetic patients. Pacing Clin Electrophysiol PACE. 2007; 30: 1267-1272. https://doi.org/10.1111/j.1540-8159.2007.00849.x PMID: 17897130

61. Akyel A, Çengel A, Tavil Y, Şahinarslan A, Topal S, Yayla Ç, et al. Relationship between plasma asymmetric dimethylarginine level and autonomic dysfunction in diabetic patients. Türk Kardiyol Derneği Arş Türk Kardiyol Derneğinin Yayın Organıdır. 2012; 40: 148-154.

62. Boer-Martins L, Figueiredo VN, Demacq C, Martins LC, Consolin-Colombo F, Figueiredo MJ, et al. Relationship of autonomic imbalance and circadian disruption with obesity and type 2 diabetes in resistant hypertensive patients. Cardiovasc Diabetol. 2011; 10: 24. https://doi.org/10.1186/1475-2840-10-24 PMID: 21426540

63. Ozturk NA, Gokturk HS, Demir M, Erdogan D, Unler GK, Gur G, et al. The effect of autonomous neuropathy on bowel preparation in type 2 diabetes mellitus. Int J Colorectal Dis. 2009; 24: 1407-1412. https:// doi.org/10.1007/s00384-009-0757-4 PMID: 19582466

64. Li X, Yu S, Chen H, Lu C, Zhang K, Li F. Cardiovascular autonomic function analysis using approximate entropy from 24-h heart rate variability and its frequency components in patients with type 2 diabetes. $\mathrm{J}$ Diabetes Investig. 2015; 6: 227-235. https://doi.org/10.1111/jdi.12270 PMID: 25802731 
65. Vukomanovic V, Tadic M, Suzic-Lazic J, Kocijancic V, Celic V. The relationship between heart rate variability and left ventricular layer-specific deformation in uncomplicated diabetic patients. Int J Cardiovasc Imaging. 2017; 33: 481-490. https://doi.org/10.1007/s10554-016-1023-9 PMID: 27853970

66. Tadic M, Vukomanovic V, Cuspidi C, Suzic-Lazic J, Stanisavljevic D, Celic V. Left atrial phasic function and heart rate variability in asymptomatic diabetic patients. Acta Diabetol. 2017; 54: 301-308. https:// doi.org/10.1007/s00592-016-0962-x PMID: 28083658

67. Adlan AM, Paton JFR, Lip GYH, Kitas GD, Fisher JP. Increased sympathetic nerve activity and reduced cardiac baroreflex sensitivity in rheumatoid arthritis. J Physiol. 2017; 595: 967-981. https://doi.org/10. 1113/JP272944 PMID: 27561790

68. Chen X, Yang R, Kuang D, Zhang L, Lv R, Huang X, et al. Heart rate variability in patients with major depression disorder during a clinical autonomic test. Psychiatry Res. 2017; 256: 207-211. https://doi. org/10.1016/j.psychres.2017.06.041 PMID: 28646783

69. Montaquila JM, Trachik BJ, Bedwell JS. Heart rate variability and vagal tone in schizophrenia: A review. J Psychiatr Res. 2015; 69: 57-66. https://doi.org/10.1016/j.jpsychires.2015.07.025 PMID: 26343595

70. Studer V, Rocchi C, Motta C, Lauretti B, Perugini J, Brambilla L, et al. Heart rate variability is differentially altered in multiple sclerosis: implications for acute, worsening and progressive disability. Mult Scle J-Exp TransI Clin. 2017; 3: 2055217317701317. https://doi.org/10.1177/2055217317701317 PMID: 28607756

71. Furlan R, Ardizzone S, Palazzolo L, Rimoldi A, Perego F, Barbic F, et al. Sympathetic overactivity in active ulcerative colitis: effects of clonidine. Am J Physiol Regul Integr Comp Physiol. 2006; 290: R224232. https://doi.org/10.1152/ajpregu.00442.2005 PMID: 16123227

72. Thorp AA, Schlaich MP. Relevance of Sympathetic Nervous System Activation in Obesity and Metabolic Syndrome. J Diabetes Res. 2015; 2015: 341583. https://doi.org/10.1155/2015/341583 PMID: 26064978

73. Liao D, Sloan RP, Cascio WE, Folsom AR, Liese AD, Evans GW, et al. Multiple metabolic syndrome is associated with lower heart rate variability. The Atherosclerosis Risk in Communities Study. Diabetes Care. 1998; 21: 2116-2122. PMID: 9839103

74. Malliani A, Montano N. Sympathetic overactivity in ischaemic heart disease. Clin Sci Lond Engl 1979. 2004; 106: 567-568. https://doi.org/10.1042/CS20040068 PMID: 15030311

75. Julius S, Nesbitt S. Sympathetic overactivity in hypertension. A moving target. Am J Hypertens. 1996; 9: 113S-120S. PMID: 8931844

76. Niedermaier ON, Smith ML, Beightol LA, Zukowska-Grojec Z, Goldstein DS, Eckberg DL. Influence of cigarette smoking on human autonomic function. Circulation. 1993; 88: 562-571. PMID: 8339419

77. Fagundes CP, Murray DM, Hwang BS, Gouin J-P, Thayer JF, Sollers JJ, et al. Sympathetic and parasympathetic activity in cancer-related fatigue: More evidence for a physiological substrate in cancer survivors. Psychoneuroendocrinology. 2011; 36: 1137-1147. https://doi.org/10.1016/j.psyneuen.2011.02. 005 PMID: 21388744

78. Stockhorst U, Huenig A, Ziegler D, Scherbaum WA. Unconditioned and conditioned effects of intravenous insulin and glucose on heart rate variability in healthy men. Physiol Behav. 2011; 103: 31-38. https://doi.org/10.1016/j.physbeh.2011.01.014 PMID: 21256144

79. Chen-Scarabelli C, Scarabelli TM. Suboptimal glycemic control, independently of QT interval duration, is associated with increased risk of ventricular arrhythmias in a high-risk population. Pacing Clin Electrophysiol PACE. 2006; 29: 9-14. https://doi.org/10.1111/j.1540-8159.2006.00298.x PMID: 16441711

80. Goit RK, Khadka R, Sharma SK, Limbu N, Paudel BH. Cardiovascular autonomic function and vibration perception threshold in type 2 diabetes mellitus. J Diabetes Complications. 2012; 26: 339-342. https:// doi.org/10.1016/j.jdiacomp.2012.03.026 PMID: 22534513

81. Huang J-C, Kuo I-C, Tsai Y-C, Lee J-J, Lim L-M, Chen S-C, et al. Heart Rate Variability Predicts Major Adverse Cardiovascular Events and Hospitalization in Maintenance Hemodialysis Patients. Kidney Blood Press Res. 2017; 42: 76-88. https://doi.org/10.1159/000469716 PMID: 28315879

82. Amra B, Behjati M, Penzel T, Fietze I, Schoebel C, Sarrafzadegan N. Nocturnal heart rate variation in diabetic and non-diabetic patients with sleep apnea syndrome. Sleep Med. 2017; 29: 57-60. https://doi. org/10.1016/j.sleep.2016.11.003 PMID: 28153217

83. Pehlivanidis AN, Athyros VG, Demitriadis DS, Papageorgiou AA, Bouloukos VJ, Kontopoulos AG. Heart rate variability after long-term treatment with atorvastatin in hypercholesterolaemic patients with or without coronary artery disease. Atherosclerosis. 2001; 157: 463-469. https://doi.org/10.1016/ S0021-9150(00)00746-2 PMID: 11472748

84. Badea AR, Nedelcu L, Valeanu M, Zdrenghea $D$. The relationship between serum lipid fractions and heart rate variability in diabetic patients with statin therapy. Clujul Med. 2014; 87: 152. https://doi.org/10. 15386/cjmed-313 PMID: 26528015 
85. Askin L, Cetin M, Turkmen S. Ambulatory blood pressure results and heart rate variability in patients with premature ventricular contractions. Clin Exp Hypertens. 2017; 1-6. https://doi.org/10.1080/ 10641963.2017.1356846 PMID: 28872359

86. de Andrade PE, do Amaral JAT, Paiva L da S, Adami F, Raimudo JZ, Valenti VE, et al. Reduction of heart rate variability in hypertensive elderly. Blood Press. 2017; 1-9. https://doi.org/10.1080/08037051. 2017.1354285 PMID: 28738697

87. Schroeder EB, Liao D, Chambless LE, Prineas RJ, Evans GW, Heiss G. Hypertension, blood pressure, and heart rate variability: the Atherosclerosis Risk in Communities (ARIC) study. Hypertens Dallas Tex 1979. 2003; 42: 1106-1111. https://doi.org/10.1161/01.HYP.0000100444.71069.73 PMID: 14581296

88. Al-Trad BA, Faris M a. IE, Al-Smadi M, Bashir A, Mansi M, Alaraj M, et al. Cardiac autonomic dysfunction in young obese males is not associated with disturbances in pituitary-thyroid axis hormones. Eur Rev Med Pharmacol Sci. 2015; 19: 1689-1695. PMID: 26004610

89. Mustafa G, Kursat FM, Ahmet T, Alparslan GF, Omer G, Sertoglu E, et al. The relationship between erythrocyte membrane fatty acid levels and cardiac autonomic function in obese children. Rev Port Cardiol Orgao Of Soc Port Cardiol Port J Cardiol Off J Port Soc Cardiol. 2017; 36: 499-508. https://doi.org/ 10.1016/j.repc.2016.10.013 PMID: 28687367

90. Yadav RL, Yadav PK, Yadav LK, Agrawal K, Sah SK, Islam MN. Association between obesity and heart rate variability indices: an intuition toward cardiac autonomic alteration-a risk of CVD. Diabetes Metab Syndr Obes Targets Ther. 2017; Volume 10: 57-64. https://doi.org/10.2147/DMSO.S123935 PMID: 28255249

91. Yoo HJ, Hwang SY, Choi KM, Baik SH, Lee EM, Kim EJ, et al. Clinical implication of body size phenotype on heart rate variability. Metabolism. 2016; 65: 1589-1596. https://doi.org/10.1016/j.metabol.2016. 08.002 PMID: 27733246

92. Dutheil F, Gordon BA, Naughton G, Crendal E, Courteix D, Chaplais E, et al. Cardiovascular risk of adipokines: a review. J Int Med Res. 2017; 300060517706578. https://doi.org/10.1177/0300060517706578 PMID: 28974138

93. Rastović M, Srdić-Galić B, Barak O, Stokić E. Association between anthropometric measures of regional fat mass and heart rate variability in obese women: Anthropometry and heart rate variability in obese women. Nutr Diet. 2017; 74: 51-60. https://doi.org/10.1111/1747-0080.12280 PMID: 28731559

94. Karason K, Mølgaard H, Wikstrand J, Sjöström L. Heart rate variability in obesity and the effect of weight loss. Am J Cardiol. 1999; 83: 1242-1247. https://doi.org/10.1016/S0002-9149(99)00066-1 PMID: 10215292

95. Reardon M, Malik M. Changes in heart rate variability with age. Pacing Clin Electrophysiol PACE. 1996; 19: 1863-1866. PMID: 8945057

96. Pavithran $\mathrm{P}$, Madanmohan T, Nandeesha H. Sex differences in short-term heart rate variability in patients with newly diagnosed essential hypertension. J Clin Hypertens Greenwich Conn. 2008; 10 : 904-910. https://doi.org/10.1111/j.1751-7176.2008.00052.x PMID: 19120716

97. LeLorier J, Grégoire G, Benhaddad A, Lapierre J, Derderian F. Discrepancies between meta-analyses and subsequent large randomized, controlled trials. N Engl J Med. 1997; 337: 536-542. https://doi.org/ 10.1056/NEJM199708213370806 PMID: 9262498 